\title{
REVIEWS
}

Check for updates

\section{Sarcomatoid renal cell carcinoma: biology, natural history and management}

Kyle A. Blum ${ }^{1}$, Sounak Gupta ${ }^{2}$, Satish K. Tickoo², Timothy A. Chan ${ }^{3}$, Paul Russo', Robert J. Motzer $\mathbb{D}^{4}$, Jose A. Karam ${ }^{5,6}$ and A. Ari Hakimi ${ }^{1,6 凶}$

Abstract | Sarcomatoid dedifferentiation is an uncommon feature that can occur in most histological subtypes of renal cell carcinomas (RCCs) and carries a decidedly poor prognosis. Historically, conventional treatments for sarcomatoid RCCs (sRCCs) have shown little efficacy, and median survival is commonly 6-13 months. Despite being first described in 1968, the mechanisms driving sarcomatoid dedifferentiation remain poorly understood, and information and treatment options available to physicians and patients are limited. When diagnosed at an early stage, surgical intervention remains the treatment of choice. However, preoperative identification through routine imaging or biopsy is unreliable and most patients present with advanced disease and systemic symptoms. For these patients, the role of cytoreductive nephrectomy is disputed. The expansion of immunotherapies approved for RCCs has generated a search for biomarkers that might be indicative of treatment response in sRCCs, although a proven effective systemic agent remains elusive. PDL1 expression is increased in sarcomatoid dedifferentiated renal tumours, which suggests that patients with sRCCs could benefit from PD1 and/or PDL1 immune checkpoint blockade therapy. Treatment outcomes for sarcomatoid tumours have remained relatively consistent compared with other RCCs, but further investigation of the tumour-immune cell microenvironment might yield insights into further therapeutic possibilities.

Renal cancers are estimated to be diagnosed in 73,750 people in the USA in 2020, and to contribute to 14,830 deaths ${ }^{1}$. The 2018 GLOBOCAN report on the global incidence of renal cancers has described 403,262 patients with renal cancer, and 175,098 deaths caused by renal cancers worldwide ${ }^{2,3}$. Approximately $90 \%$ of all diagnosed renal parenchymal malignancies are renal cell carcinomas (RCCs) ${ }^{4}$.

A rare transformation called sarcomatoid dedifferentiation can occur in most RCC histological subtypes and portends an especially poor prognosis. RCCs in which sarcomatoid dedifferentiation has occurred are commonly referred to as sarcomatoid RCCs (sRCCs), and patients with sRCCs often present with advanced or metastatic disease and rarely survive $>1$ year ${ }^{5-7}$. Sarcomatoid features are present in approximately $4-5 \%$ of all $\mathrm{RCCs}^{8-11}$. However, this proportion can range from 1 to $29 \%$ depending on the primary histology and the reporting study ${ }^{7,9-13}$. Although infrequently diagnosed in the localized setting ( 20-40\% of all sRCCs $)^{14,15}$, approximately $20 \%$ of patients with metastatic RCC harbour sarcomatoid dedifferentiation ${ }^{15}$. Patients commonly present with an sRCC at between 54 and 63 years of age $^{6,7,13,14,16,17}$, and the male-to-female ratio ranges from 1.3:1 to $2: 1$ (REFS $^{6,13,14,18,19}$ ). Similar to all other RCCs, the mechanisms underlying this gender difference remain unclear. However, possibilities include historical gender differences in occupational exposure or smoking habits, or the influence of sex hormones on tumour biology $\mathrm{y}^{20}$.

The natural history and prognosis of sRCCs are poor, as approximately $60-80 \%$ of patients present with advanced or late-stage disease ${ }^{14,15}$. Median survival is approximately 6-13 months and a higher percentage of sarcomatoid dedifferentiation on histology has been reported to confer a worse prognosis ${ }^{6,711,21,22}$. Retrospective reports have shown reduced overall survival and cancer-specific survival in patients with increased sarcomatoid features ${ }^{11,21}$. Compared with patients without sarcomatoid features, patients with $\geq 25 \%$ sarcomatoid features had $\sim 30 \%$ increased overall risk of dying of any cause (HR 2.07, $P=0.048)^{11}$ and those with $\geq 30 \%$ sarcomatoid features had a $52 \%$ increased risk of 


\author{
Key Points \\ - Sarcomatoid dedifferentiation is not considered to be a unique histological subtype \\ of renal cell carcinomas (RCCs); rather, it can be present within any subtype of RCCs. \\ - Sarcomatoid dedifferentiation appears in $\sim 4 \%$ of all RCCs, but is present in $\sim 20 \%$ \\ of all metastatic RCCs. According to WHO guidelines, any RCC with sarcomatoid \\ dedifferentiation is a WHO-International Society of Urological Pathology \\ grade 4 lesion. \\ - Sarcomatoid dedifferentiation is often heterogeneously present within RCCs, making \\ routine imaging and biopsy unreliable for preoperative detection. Surgical resection \\ for localized disease is the standard of care, with subsequent close monitoring of \\ patients following surgery. \\ - In patients with metastatic disease, conventional therapies such as surgery and \\ systemic agents have been ineffective and overall 5 -year survival remains at \\ $23.5-33 \%$. \\ - Previous genomic analyses have failed to identify definitive mutational drivers \\ of disease. However, sarcomatoid RCCs (sRCCs) have been shown to have higher \\ PD1 and PDL1 expression than other subtypes of RCCs. Newer combinations of \\ immune checkpoint inhibitor immunotherapies could yield improved responses \\ and outcomes. \\ - Studies investigating sRCCs are limited by patient numbers owing to the low \\ incidence of sRCCs and their advanced stage at presentation. Multi-institutional \\ efforts to establish a consensus on treatment recommendations based on highly \\ powered data are essential.
}

treatment options and is likely a result of limited research availability, as sRCC is a rare form of RCC that is often at an advanced stage by the time of presentation.

In this Review, we discuss and consolidate the current knowledge on sRCCs. We first explore the origins of sRCC nomenclature to assist in the understanding of current naming conventions. We then review the common presentation of sRCCs and modern techniques for pathological diagnosis. We summarize contemporary knowledge on the molecular biology of sRCC, including next-generation genomic sequencing investigations and their findings. Finally, we discuss treatment options including surgery, radiotherapy and systemic therapy and summarize ongoing studies and recommendations.

\section{History of sRCC}

In 1943, Weisel et al. ${ }^{27}$ described renal tumours with sarcoma-like appearance. Pathologists later reported subsets of renal sarcomas with characteristics of RCCs that were clinically more aggressive than conventional $\mathrm{RCCs}^{28}$. The term "carcinosarcoma of the kidney" was used to distinguish these variants from both renal soft-tissue sarcoma and conventional RCCs ${ }^{28}$. In 1968, Farrow and colleagues ${ }^{29}$ coined the term "sarcomatoid renal cell carcinoma" to describe a renal malignancy closely resembling a sarcoma with pleomorphic spindle-cell and giant-cell morphology. The authors wrote that sarcomatoid RCC seemed to be "a complex tumour of an altogether different type" and that its appearance was similar to "that of a double neoplasm, one component of which is a [renal cell] carcinoma of the adult type and the other an undifferentiated pleomorphic [tumour] which appears morphologically sarcomatous" 29 . Subsequently, sRCC was considered to be a separate histology as its clinical behaviour was more aggressive than that of a conventional RCC ${ }^{29}$. However, the labelling of an sRCC as a distinct histological entity came under scrutiny, as pathologists recognized that sarcomatoid features could be found in conjunction with most histological subtypes of RCC. Work by Thoenes and colleagues published in 1986 resulted in the Mainz classification of renal tumours, echoing contemporary sentiments that the "sarcomatous" variant "in principle may be related to most cell types" and proposed sRCC as an end-stage dedifferentiated tumour ${ }^{30}$. By 1997, the American Joint Committee on Cancer (AJCC) ${ }^{31}$, Union Internationale Contre Le Cancer (UICC) ${ }^{31}$ and the Heidelberg classification of renal cell tumours ${ }^{32}$ all formally removed sRCC as a distinct histology and assessed it to be a feature that could be present within any histology of RCC. Following these events, pathologists met to standardize criteria for diagnosing and reporting sRCCs. In 2012, the International Society of Urological Pathology (ISUP) Consensus Conference established that sarcomatoid transformation could be designated within an RCC if it contained atypical spindle cells and resembled "any form of sarcoma" 33 . No minimum amount or percentage of sarcomatoid dedifferentiation is required to establish the diagnosis of sRCC $^{33}$. The 2012 Consensus Conference also updated the definition of grade to include RCC with sarcomatoid dedifferentiation in the category of WHO-ISUP 
grade 4 and mandated that the background epithelial RCC histology must be reported (e.g. papillary RCC with sarcomatoid features). Finally, it was determined that RCCs that contained $\sim 100 \%$ sarcomatoid dedifferentiation with no recognizable epithelial RCC component should be labelled unclassified RCCs (uRCCs), WHO-ISUP grade 4 . These 2012 reporting recommendations were officially endorsed in 2016 by the World Health Organization in their genitourinary classification guidelines ${ }^{34}$, aiding in the standardization of reporting techniques for sRCC. Now, although sRCC is not considered its own histology within RCC, the terms sarcomatoid features, sarcomatoid elements, sarcomatoid dedifferentiation, sarcomatoid differentiation, sarcomatoid component and sarcomatoid renal cell carcinoma are often used interchangeably to describe the same entity, namely sRCC.

\section{Presentation \\ Clinical presentation}

The presentation of sRCCs varies and depends on the stage of disease at diagnosis. Most patients present with locally advanced or metastatic disease and, therefore, approximately $90 \%$ are symptomatic at presentation ${ }^{7,10,17,19}$. Signs and symptoms are routinely non-specific and can consist of flank or abdominal pain (present in $51 \%$ of symptomatic patients), haematuria (22-34.7\%), weight loss (18-22.6\%), fatigue (15\%), fever $(6-10.6 \%)$, night sweats $(6-12.6 \%)$ and cough and/or dyspnoea $(6 \%)^{7,10,19,26}$. The most common sites for distant metastasis, in decreasing order of prevalence, include the lungs (34.6-71.0\%), bone (13.0-44.0\%), lymph nodes $(25 \%)$, liver (12.6-23.0\%) and brain $(5.1-16.0 \%)^{14,15,17,24,35,36}$.

\section{Radiographic presentation}

To date, reliable preoperative imaging methods capable of identifying sarcomatoid dedifferentiation within a renal tumour do not exist ${ }^{15,37}$. Specific biomarkers for sRCCs have yet to be described and biopsy accurately detects sarcomatoid features within tumours at a low rate, $\sim 7.5 \%{ }^{19}$. Retrospective studies have attempted to elucidate features on CT and MRI imaging that would help diagnose sRCCs preoperatively. Unfortunately, these data are sparse and in the past 5 years only one study has investigated sarcomatoid features in CT imaging - the most commonly ordered imaging modality used in the radiographic assessment of renal masses.

A retrospective case-control study assessing preoperative CT images with texture analysis in $\operatorname{sRCC}(n=20)$ versus ccRCC $(n=25)$, found that sRCCs were on average larger than ccRCCs, measuring $7.7 \mathrm{~cm}$ compared with $5.0 \mathrm{~cm}(P=0.003)$, respectively ${ }^{38}$. Other features more commonly associated with sRCCs than ccRCCs included peritumoural neovascularity $(P=0.001)$, larger peritumoural vessels $(P<0.001)$ and a more heterogeneous texture analysis $(P<0.001)$. However, these findings are non-specific ${ }^{38}$.

On preoperative MRI, sRCC has been reported to have similar non-specific and subtle differences from ccRCC on T2-weighted images, such as more irregular and infiltrative morphology $y^{39,40}$. One study assessed 11 patients with pathologically confirmed ccRCCs with sarcomatoid dedifferentiation. Patients underwent preoperative MRI, the results of which were studied to identify differences between the sarcomatoid and epithelial tumour components. Using normal renal cortex (defined as T2 low-intensity signal) as a baseline, the investigators found that sRCCs had a more hypointense signal than ccRCCs, which had an isointense-to-high signal intensity ${ }^{40,41}$. Building on their previous study, the researchers applied a scoring system between 1 and 5 based on the presence of low-intensity signals within the tumour in a cohort of renal masses (10 sRCCs and 131 non-sarcomatoid masses). With a positive predictive value of $56 \%$ and negative predictive value of $99 \%$, low-intensity areas in renal tumours of $1-3 \mathrm{~cm}$ were considered probable for sRCCs and tumours with low-intensity areas of $>3 \mathrm{~cm}$, multiple $>1$-cm low-intensity areas, or disruption of the pseudocapsule were considered definite sRCC findings ${ }^{41}$. This classification study ${ }^{41}$ has served as the basis for relative thresholds used in subsequent work ${ }^{42}$ to calculate the percentage volume of sarcomatoid involvement in RCC tumours on MRI. Sarcomatoid percentage estimated by MRI showed a positive Pearson correlation with the post-nephrectomy histology $(\mathrm{R}=0.782, P<0.001)^{42}$. However, further analysis revealed poor agreement with the histological examination, as MRI estimations underestimated percentage sarcomatoid values by at least $19 \%{ }^{42}$.

PET-CT with ${ }^{18} \mathrm{~F}$-fluorodeoxyglucose $\left({ }^{18} \mathrm{~F}-\mathrm{FDG}\right)$ for detection of sRCCs has been previously described in four separate case reports ${ }^{43-46}$ to assess for recurrence, metastasis or initial staging ${ }^{43}$. However, in larger conventional RCC series ${ }^{18} \mathrm{~F}$-FDG PET/CT has not demonstrated clinical usefulness ${ }^{47}$, and current professional practice guidelines from the American Urological Association $^{48}$, European Society for Medical Oncology ${ }^{49}$ and the European Association of Urology $y^{50}$ do not routinely recommend its use for diagnosing or staging RCC. Currently, the utility of ${ }^{18} \mathrm{~F}-\mathrm{FDG}-\mathrm{PET} / \mathrm{CT}$ in the diagnosis and management of sRCC is questionable. Most patients with sRCC present with gross metastatic disease, which can be effectively viewed using CT and MRI. Although studies are ongoing, the ability of CT and MRI to preoperatively identify sRCCs or the percentage of sarcomatoid involvement within a tumour remains unreliable for clinical decision-making at this time and at best underestimates the true extent of sarcomatoid disease $\mathrm{e}^{42}$. Further research to identify radiographic features of sRCC is needed.

\section{Pathology}

sRCC is not a distinct histological subtype of RCCs but is a pattern of dedifferentiation characterized by the loss of epithelial features that are classic to $\mathrm{RCCs}^{34}$. Sarcomatoid dedifferentiation can be present within most histologies of RCC ${ }^{31,33,34}$, although it is most commonly seen in ccRCCs and chromophobe RCCs ${ }^{10,35}$. However, current incidence data are primarily based on smaller series $(<150 \text { patients })^{10,11,16}$, which require validation in larger datasets. 


\section{Histological diagnosis}

sRCCs are often large (median $\sim 10 \mathrm{~cm}$ ) and can appear as dense grey or white areas within the tumour architecture that usually reveal a firm and fleshy cut surface when dissected ${ }^{6,18,51}$ (FIG. 1). The microscopic features of sRCCs often include a mixture of both epithelial and sarcomatoid components (FIG. 2). Unlike classic RCCs, the sarcomatoid component does not have recognizable epithelial components ${ }^{10}$ and histologically appears similar to sarcomas with pleomorphic and spindle cells with high cellularity and atypia ${ }^{52}$. These regions of sarcomatoid dedifferentiation can be heterogeneous or uniform. Approximately $90 \%$ of sRCC cases have coagulative necrosis, and roughly $30 \%$ contain some form of microvascular invasion ${ }^{6,7,10}$. Rhabdoid features are reported in approximately $15 \%$ of sRCC tumours ${ }^{53}$. According to the 2016 WHO guidelines, the presence of any amount of sarcomatoid dedifferentiation is sufficient for diagnosis of RCC with sarcomatoid features ${ }^{33,34}$.

Sarcomatoid dedifferentiation has been described in roughly $5-8 \%$ of ccRCCs, $8-9 \%$ of chromophobe RCCs, $2-3 \%$ of papillary RCCs, $2-3 \%$ of uRCCs and up to $29 \%$ of collecting-duct RCCs ${ }^{6,10}$. Overall, the most common histology seen with sarcomatoid features is ccRCC, which comprises $\sim 80 \%$ of all RCC cases $^{54}$. Reports have suggested that chromophobe RCCs, which comprise $\sim 5-10 \%$ of all RCC cases $^{55}$, might harbour a higher rate of sarcomatoid dedifferentiation than $\mathrm{ccRCCs}^{10,56}$, although the higher prevalence of ccRCCs made it difficult to validate this finding. Sarcomatoid change has also been reported in acquired cystic disease $\mathrm{RCCs}^{57}$ and hereditary leiomyomatosis RCC syndrome-associated RCCs $^{58,59}$.

Diagnostic sampling of the primary tumour is recommended, as the well-differentiated epithelial component and dedifferentiated sarcomatoid component might only be focally present, which could lead to underdiagnosis.

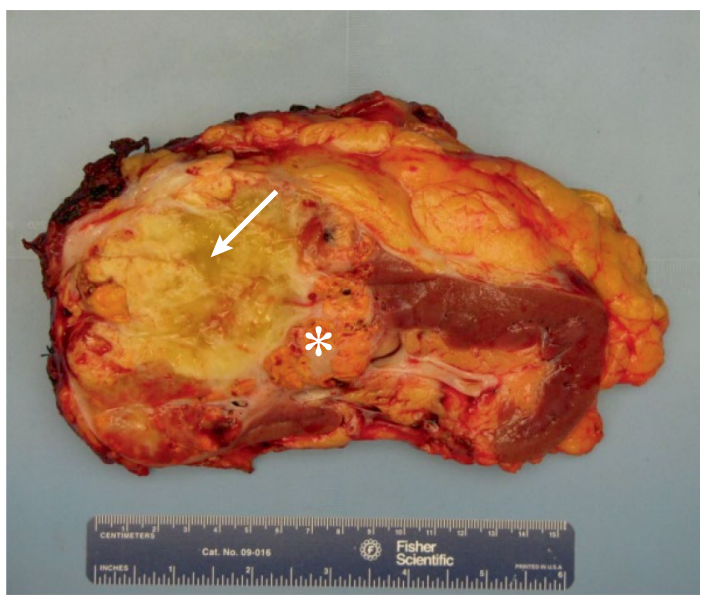

Fig. 1 | Gross sections of an sRCC after radical nephrectomy. Sarcomatoid renal cell carcinoma (sRCC) components are often large and can appear as dense grey or white areas within the tumour architecture and typically reveal a firm and fleshy cut surface when dissected. Arrow shows fleshy area that corresponds to sarcomatoid transformation and asterisk marks yellow and friable area of the tumour that corresponds to lower grade clear cell renal cell carcinoma.
Immunohistochemical markers, including markers of renal histogenesis, can help to resolve this diagnostic dilemma ${ }^{60}$. In the absence of a low-grade epithelial component such markers are useful, as a tumour with spindled morphology has a wide differential diagnosis that includes sarcomatoid urothelial carcinoma, angiomyolipoma, dedifferentiated liposarcoma, sarcomatoid adrenocortical carcinoma, and mesenchymal neoplasms such as solitary fibrous tumour and synovial sarcoma, and others ${ }^{6}$. Thus, a diagnosis of sRCC relies heavily on the use of ancillary testing to establish renal histogenesis in the form of immunohistochemistry for transcription factors such as PAX2 and PAX8, which are both nephric-lineage transcription factors required for establishing renal-lineage cells and kidney formation and, therefore, a useful maker for renal epithelial tumours ${ }^{61,62}$. Additionally, markers of epithelial differentiation including pan-cytokeratins and/or epithelia membrane antigen can be employed, which are often focally expressed in the sarcomatoid component of RCCs but are negative in sarcomas. Finally, markers specific to underlying epithelial subtypes, such as carbonic anhydrase IX for clear cell RCCs, can also be employed ${ }^{63}$ (FIC. 2).

\section{Role of biopsy}

The use of percutaneous renal biopsy for suspicious masses has increased since the early $2000 \mathrm{~s}^{64}$. Although percutaneous biopsies have shown satisfactory correlation with histological classification at the time of nephrectomy, determining the presence of sarcomatoid dedifferentiation can be difficult ${ }^{37,65}$. Identifying sarcomatoid features is limited primarily by the amount of tissue obtained by core biopsy and by the number and spatial distribution of biopsy cores. Sarcomatoid features often comprise $<50 \%$ of sRCC tumours, which are commonly large heterogeneous masses ${ }^{15}$. In a study of 199 patients with an sRCC, biopsies of the primary tumour or metastatic site prior to nephrectomy or metastasectomy detected the presence of sarcomatoid features in $7.5 \%$ of patients ${ }^{19}$. Thus, the sensitivity of preoperative biopsies for sRCCs is low and is likely non-diagnostic for these features. Multi-quadrant biopsies have shown improved sensitivity in diagnosing sRCCs ${ }^{66}$. In a study comparing standard single biopsy with multi-quadrant biopsy (biopsies from at least four separate solid enhancing areas in the tumour), 23 of 96 large RCCs studied on post-nephrectomy final pathology were sRCCs. Sensitivity for identifying sarcomatoid features was higher using the multi-quadrant technique than the standard biopsy technique (86.7\% versus $25.0 \%$, $P=0.0062)^{66}$. To date, the most reliable method for diagnosing sRCC is based on post-surgical final pathological review by a dedicated genitourinary pathologist.

\section{Metastases}

Metastases of sRCCs can exhibit sarcomatoid features similar to those in the primary tumour. Information on the differences between sarcomatoid and nonsarcomatoid metastasis from sRCC is limited. However, patients with $>30 \%$ sarcomatoid dedifferentiation within their primary tumour frequently have sarcomatoid features in their metastases?. 

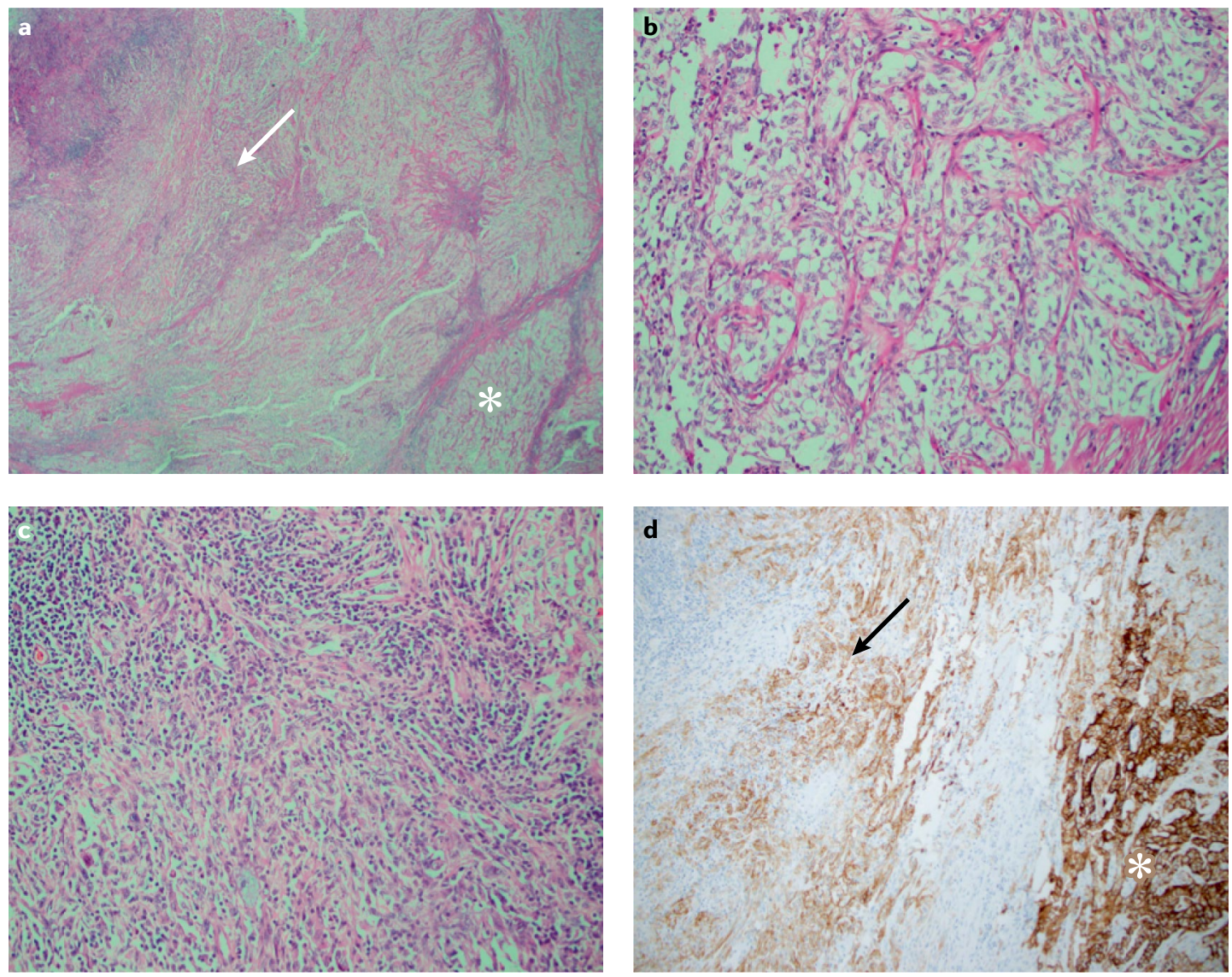

Fig. 2 | Histopathology of sRCC. Part a ( $\times 40$ magnification) shows a representative area of a sarcomatoid renal cell carcinoma (sRCC) with a well-differentiated clear cell carcinoma (ccRCC) component (asterisk) and a dedifferentiated sarcomatoid component (arrow). Magnified areas of the ccRCC (part b, $\times 200$ magnification) and dedifferentiated sarcomatoid components (part c, $\times 200$ magnification) are also shown. The ccRCC comprises cells with optically clear cytoplasm organized in alveolar/acinar architectural patterns, whereas the sarcomatoid component exhibits spindled cells associated with a dense lymphocytic infiltrate. Immunohistochemistry for carbonic anhydrase IX ${ }^{192}$ (a HIF-1 $\alpha$ target gene that shows diffuse membranous localization in ccRCCs, which have increased HIF-1a signalling secondary to alterations of $\mathrm{VHL}$ ) shows an area of transformation (part $\mathbf{d}, \times 40$ magnification), with strong cell membrane-localized expression in the well-differentiated ccRCC areas (asterisk) and gradual loss of expression in the more poorly differentiated sarcomatoid areas (arrow).

\section{Tumour biology}

An sRCC is composed of two separate cell types, the sarcomatoid (mesenchymal) component, and the RCC (epithelial) component. The mechanism by which sarcomatoid dedifferentiation arises within RCCs is not clearly understood; however, evidence suggests that the sarcomatoid component might originate from a common cell-of-origin, resulting in cells that lose their epithelial characteristics and gain mesenchymal characteristics in a process known as epithelial-mesenchymal transition (EMT) $)^{5,67-70}$. EMT is an essential transformation process in early development to generate layers of specialized tissue. However, EMT can also contribute to tumorigenesis ${ }^{71}$. EMT is regulated by a group of transcription factors including Snail, Zeb and Twist, which cause hallmark downregulation of epithelial markers such as E-cadherin and upregulation of mesenchymal markers such as $\mathrm{N}$-cadherin ${ }^{60,72}$ (FIG. 3). E-cadherin is a cell membrane protein important in cell-cell adhesion and is attached to the cytoskeleton via the protein $\beta$-catenin. During EMT, $\beta$-catenin translocates to the nucleus where it acts as a transcription factor for Snail, a transcriptional repressor of E-cadherin ${ }^{71,73-76}$. As a result, epithelial cells lose their phenotypic features (such as expression of E-cadherin) and subsequently gain mesenchymal characteristics, leading to an increased ability to metastasize ${ }^{77}$, which might contribute to the aggressive features of sRCCs $^{60}$ (FIG. 3). Evidence of EMT in sRCC was reported in a study of 21 tumours containing matched epithelial and sarcomatoid components immunohistochemically examined for known markers of EMT ${ }^{68,78}$. In the sarcomatoid components, expression of E-cadherin was reduced and expression of $\mathrm{N}$-cadherin, Snail and $\beta$-catenin was increased ${ }^{68}$. Notably, E-cadherin and $\mathrm{N}$-cadherin were both found to be highly expressed in the RCC (epithelial) components; however, the physical localization of cadherin in the cell varied. In the sarcomatoid component, $\mathrm{N}$-cadherin was predominantly present in the cytoplasm and, in the epithelial component, $\mathrm{N}$-cadherin was primarily localized to the cell membrane. Subcellular localization to the cell membrane is 


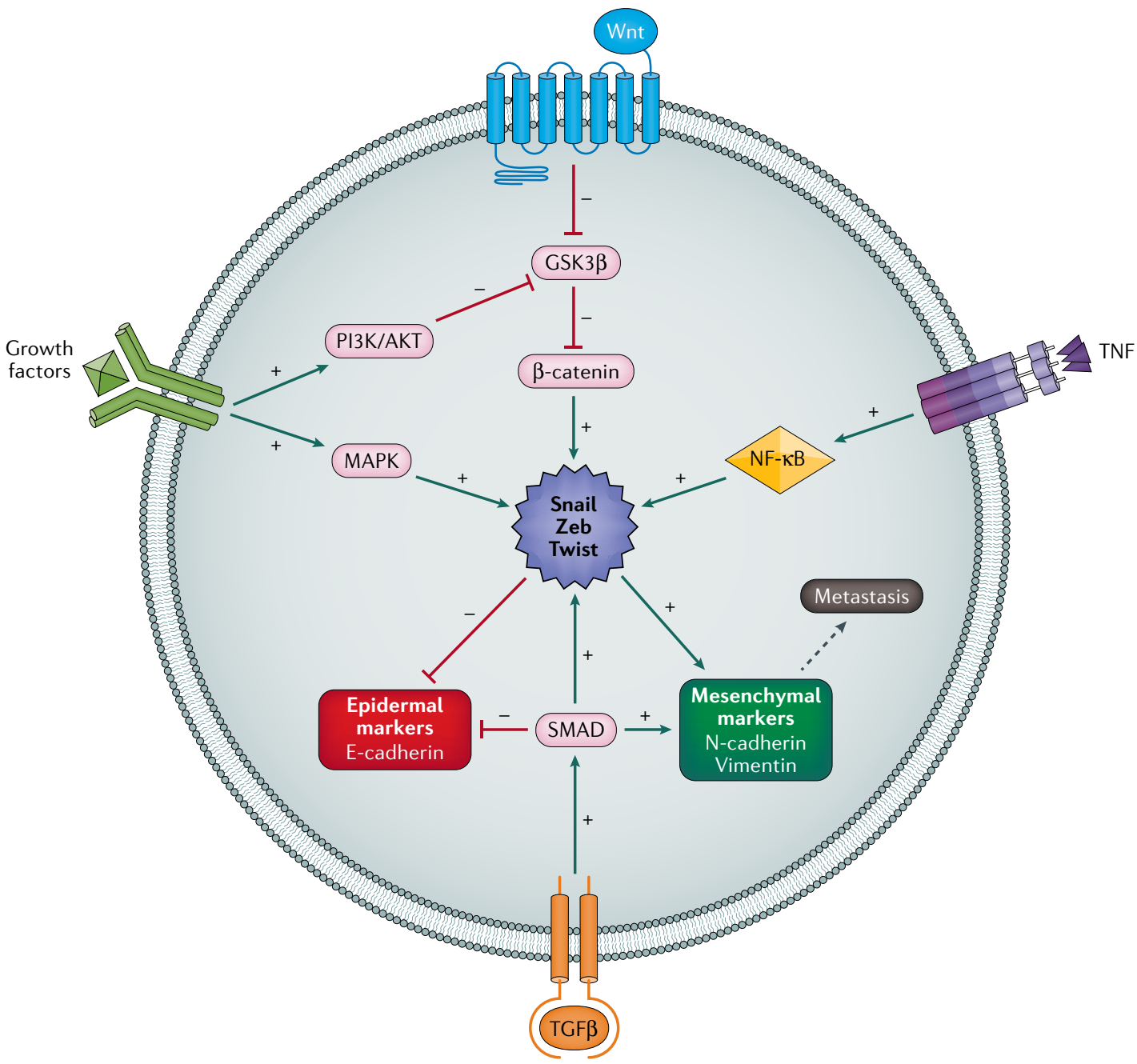

Fig. 3 | Signalling pathways involved in EMT reported in sRCC. A sarcomatoid renal cell carcinoma (sRCC) is composed of two separate cell types, the sarcomatoid (mesenchymal) component, and the RCC (epithelial) component. The mechanism by which sarcomatoid dedifferentiation arises within RCC is not clearly understood; however, there is evidence that the sarcomatoid component may originate from a common cell-of-origin, resulting in cells that lose their epithelial characteristics and gain mesenchymal characteristics through a process called epithelial-mesenchymal transition (EMT). EMT can occur via multiple pathways including TNF, TGF $\beta$, Wnt, MAPK and PI3K/AKT signalling to regulate expression of Snail, Zeb and Twist. Activation of these transcription factors results in the downregulation of epithelial markers (E-cadherin) and upregulation of mesenchymal markers (N-cadherin). E-cadherin is a cell membrane protein that is important in cell-cell adhesion; however, during EMT these intercellular tight junctions (E-cadherin) break down and transform the cell into a more mesenchymal phenotype that increases the likelihood of tumour cell metastasis.

consistent with epithelial cell-cell adhesion, whereas the movement of $\mathrm{N}$-cadherin into the cytoplasm promotes cell motility ${ }^{79,80}$. The authors of this study on EMT in sRCCs ${ }^{68}$ conclude that these results provide strong evidence for sRCC as an example of EMT and support the leading theory that sRCC originates from a precursor epithelial component that has undergone EMT. Indeed, sRCC is considered a classic example of a mesenchymal phenotype rationalized by EMT, with multiple studies supporting this theory $\mathrm{y}^{5,67-70,78,81,82}$. Moreover, in vitro investigations into sRCC cell lines have shown that the mesenchymal markers vimentin and $\mathrm{N}$-cadherin are strongly expressed in tumour cell cytoplasm, with minimal expression of intracytoplasmic E-cadherin, providing further evidence that sRCC biology includes EMT $^{82}$.
In addition to genomic alterations, EMT can be related to epigenetic regulatory mechanisms such as methylation of gene promoters, histone modifications or microRNA-induced expression changes ${ }^{83-86}$. Studies on epigenetic regulatory mechanisms specific to sRCCs are not yet available, although these mechanisms have been reported in RCCs ${ }^{83,84,87}$. In a 2010 study that used clinical tumour samples and xenograft models to assess genomics and promoter methylation in the establishment of RCC metastasis ${ }^{83}$, variations in methylation contributed to the expression of pro-metastatic mesenchymal genes in non-metastatic RCC cells. In non-metastatic RCCs, epigenetically silenced genes were identified via demethylation using DNA methyltransferase inhibitor $5^{\prime}$-AZA, which leads to upregulation of pro-mesenchymal genes such as $S 100 A 4^{88,89}$ - a known 
fibroblast marker that mediates metastasis and EMT $^{88}$. Without $5^{\prime}$-AZA treatment, pro-mesenchymal S100A4 remained methylated (downregulated) in non-metastatic RCC cells ${ }^{83}$. By contrast, S100A4 in metastatic RCC cells was hypomethylated and thus spontaneously upregulated at baseline. These results suggest that epigenetic mechanisms can lead to a mesenchymal phenotype in RCCs that enables tumour progression. Indeed, the impact of promoter methylation on EMT is not unprecedented, as altered methylation of gene promoters has resulted in EMT tumour progression in breast cancer ${ }^{86}$. Such epigenetic mechanisms may contribute to the aggressive mesenchymal phenotype $\mathrm{e}^{68,78}$ observed in sRCCs. Evidence of these epigenetic mechanisms was identified in a 2013 study that characterized an sRCC cell line for cancer modelling ${ }^{82}$, in which sRCC cells were found to be positive for cytoplasmic N-cadherin, vimentin and S100A4. Notably, S100A4 was present in the cytoplasm in $52.7 \%$ of sRCC cells on cytofluorometric analysis, and vimentin and $\mathrm{N}$-cadherin were expressed in the cytoplasm in $99.8 \%$ and $81.2 \%$ of sRCC cells, respectively. S100A4 is an example of a gene integral to EMT (and metastasis) in RCC that is regulated through methylation modulation. Thus, it might also be possible that the same epigenetic mechanisms could be exploited for the methylation or demethylation of corresponding promoter sequences of interest as a future treatment in sRCC.

\section{Framework for genomic investigation}

Before the advent of next-generation sequencing only a few studies examined genomic aberrations present in sRCC. Four notable studies ${ }^{63,68,69,90}$ have created a foundation for modern genomic analysis of sRCC.

In 1995, Oda et al. ${ }^{90}$ compared matched epithelial and sarcomatoid components in 14 tumour samples and used PCR to assess the mutational status of TP53 and HRAS, both of which are known to be associated with cancer progression. Although no HRAS mutations were observed, $78.6 \%$ of tumours had TP53 mutations within their sarcomatoid component, and only $14.3 \%$ of tumours had TP53 mutations in their epithelial component. Furthermore, p53 overexpression was observed on qualitative immunohistochemical analysis in sarcomatoid components compared with their corresponding epithelial components. The authors concluded that TP53 mutation likely results in overexpression of mutated p53 and is critical for sarcomatoid transformation ${ }^{90}$.

In 2005 , Jones et al.$^{69}$ conducted a study of 22 patients with sRCC cases to assess the molecular and clonal relationship between matched ccRCC epithelial and sarcomatoid components in laser-microdissected tumours. $\mathrm{X}$ chromosome inactivation and loss of heterozygosity $(\mathrm{LOH})$ at loci previously implicated in RCCs (3p14, $7 \mathrm{q} 31,8 \mathrm{p} 21,9 \mathrm{p} 21$, and $17 \mathrm{p} 13$ ) were analysed ${ }^{69}$. Patterns of allelic loss were variable in both epithelial and sarcomatoid components, highlighting substantial genetic heterogeneity within individual RCCs ${ }^{69}$. In 13 of 14 female patients, the same pattern of X chromosome inactivation was observed in matched epithelial and sarcomatoid components, supporting a clonal cell-of-origin theory ${ }^{69}$.
In 2007, Tickoo et al. ${ }^{63}$ assessed the expression of hypoxia inducible factor-1a (HIF1 $\alpha$ ), glucose transporter 1 (GLUT1), carbonic anhydrase IX (CAIX), and vascular endothelial growth factor (VEGF) in 34 RCCs with sarcomatoid dedifferentiation (22 ccRCCs and 12 non-ccRCCs) using immunohistochemistry. Loss of the von Hippel-Lindau ( $V H L)$ tumour suppressor gene is a defining feature of ccRCCs ${ }^{91,92}$. The VHL protein assists in the activation of a E3-ubiquitin complex responsible for degrading HIF. In the presence of oxygen normotension, HIF is marked for ubiquitylation. In cells with low oxygen tension or loss of functional VHL (as seen in ccRCCs), HIF escapes degradation, and activates downstream targets that assist in tumorigenesis, including VEGF and GLUT1 (REF. ${ }^{63}$ ). In the study by Tickoo et al. ${ }^{63}$, sarcomatoid dedifferentiation in ccRCCs was associated with higher levels of HIF than sarcomatoid dedifferentiation in non-ccRCCs, suggesting that sarcomatoid components maintain some similarity to their primary histology.

In 2011, Conant et al. ${ }^{68}$ examined known markers of EMT in matched epithelial and sarcomatoid components in 21 sRCCs. Within the sarcomatoid component, a loss of epithelial markers and a gain of mesenchymal markers were observed, further supporting EMT as a mechanism for transformation to $\mathrm{sRCC}^{68}$.

\section{Next-generation sequencing}

The use of next-generation sequencing to evaluate the genomics of sarcomatoid transformation has provided additional evidence of the common cell-of-origin theory ${ }^{93-97}$.

In 2016, Bi et al. ${ }^{93}$ used whole-exome sequencing to examine sarcomatoid dedifferentiation in $21 \mathrm{ccRCCs}$ with epithelial, sarcomatoid and normal kidney components. Two hypermutated tumours suspected of being biologically different were excluded from analysis. Across the remaining 19 tumours, $41.7 \%$ of somatic single-nucleotide variants (SSNVs) were shared between epithelial and sarcomatoid components. The most frequently shared SSNVs were in VHL, PBRM1, PTEN and SETD2. TP53 was the most commonly mutated gene in sarcomatoid components: mutations were present in the sarcomatoid element of $31.6 \%$ of tumours, and not present in any of the matched epithelial components. In addition, some sarcomatoid components had increased expression of cancer driver genes such as BAP1 (10.5\%) and ARIDIA (15.7\%), which were mutually exclusive with TP53 and with each other. Furthermore, several novel SSNVs were reported in the sarcomatoid component, including mutations in FAT1, FAT2 and FAT3, TSG101, LRIF1, RQCD1 and PTK7 (REF. ${ }^{93}$ ). Overall, the sarcomatoid component had a higher mutational burden than the epithelial component and sarcomatoid-specific LOH on chromosomes $1 \mathrm{p}, 9,10,14,17 \mathrm{p}, 18$ and 22 (REF $\left.{ }^{93}\right)$. This study was one of the first to suggest - using three major findings - that sarcomatoid components in sRCCs originated from a pre-existing epithelial component through a process of dedifferentiation. First, the authors showed that the most frequently mutated genes shared between the epithelial and sarcomatoid components were those commonly mutated in ccRCCs. 
Second, the frequency of SSNVs in known cancer driver genes was greater than five times higher in the sarcomatoid versus the epithelial regions. Last, mutation of TP53 was more common in sarcomatoid elements than in non-sarcomatoid elements, suggesting a specific role for TP53 in the development of sarcomatoid elements, in addition to recurrent mutations and/or segments of $\mathrm{LOH}$ affecting other known cancer genes. These findings lend support to a pathogenic sequence theory in which somatic mutations that occur in ccRCC drive dedifferentiation to a sarcomatoid state.

Malouf et al. ${ }^{94}$ also sought to identify genomic alterations in sRCC in a 2016 study. In addition to ccRCC, papillary, collecting duct and uRCC primary histologies were included for analysis. In their study, 26 patients with sRCCs in three unique cohorts underwent genomic profiling. The first cohort comprised three sarcomatoid ccRCCs from three patients with matched microdissected epithelial and sarcomatoid components who underwent targeted sequencing using a panel of 236 frequently mutated cancer-related genes and 37 introns frequently rearranged in cancer. Two tumours showed identical mutational profiles between their epithelial and sarcomatoid components. However, the third tumour had distinct inactivating mutations of PTEN and TP53 between the epithelial and sarcomatoid components, and JAK2 was amplified in the sarcomatoid component. In the second cohort, 26 sRCC tumours from 26 patients (23 patients plus the previous 3 ) associated with various primary histologies were analysed. The most frequently mutated gene was TP53 (in $42.3 \%$ of tumours), followed by CDKN2A (26.9\%) and NF2 (19.2\%). In the final cohort, whole-exome sequencing was performed on four non-microdissected tumours from four patients with sarcomatoid ccRCCs. Median mutation rate was lower in these four sarcomatoid ccRCCs than in The Cancer Genome Atlas downloaded dataset of $446 \mathrm{ccRCCs}^{98}$ 37.5 vs 49 mutations per case, respectively. Additionally, Sanger sequencing of $V H L$ and TP53 in multiple regions from the primary tumours of two patients did not show intratumoural heterogeneity for these two genes.

A subsequent study that included 252 sRCCs from a total dataset of 2,636 RCCs supported the findings of Malouf et al. ${ }^{94}$ by showing increased prevalence of JAK2 (9p24.1) amplifications in sRCCs: JAK2 amplifications were present in $5.95 \%$ of sRCCs compared with $0.6 \%$ of all RCC tumours $(P<0.001)^{99}$. Additionally, co-amplified genes at the 9p24.1 locus included PDL1 and $P D L 2$, and these amplification events correlated with immunohistochemistry-based programmed cell death 1 ligand 1 (PDL1) expression (mean $H$ score: $222 / 300, n=10)$. Amplification of PDL1 and PDL2 was often absent in low-grade epithelial components and restricted to the sarcomatoid tumour component ${ }^{99}$ (FIG. 4). Gupta et al. ${ }^{99}$ documented the presence of constitutive PDL1 expression primarily in high-grade components, although the exact frequency was not documented owing to the study design (scoring of tissue microarrays of high-grade RCCs). However, these findings were supported by the observations of Kawakami et al. ${ }^{100}$

Wang et al. assessed genomic and molecular characteristics of 40 frozen sRCCs with papillary, chromophobe and clear cell histologies through unsupervised clustering analysis of copy number and transcriptional data, and showed that sarcomatoid dedifferentiation segregated according to primary histology (clear cell, chromophobe, papillary) rather than to epithelial or sarcomatoid morphological components ${ }^{96}$. This finding suggests that sRCCs can be classified by primary histology instead of being labelled as a broad disease category of sRCCs. Whole-exome, single-nucleotide polymorphism characterization and RNA sequencing, showed that the overall mutational burden of sRCCs was similar to that of RCCs ${ }^{96}$. Gene mutations shared across all sRCC samples included VHL, C10orf113, BAP1, TMEM97, CALML3, IL15 and, most notably, PTEN, TP53, NF2 and RELN. When analysing specific histologies, PTEN was more frequently mutated in sarcomatoid ccRCCs than in ccRCCs, TP53 mutations were elevated in sarcomatoid chromophobes and ccRCCs compared with their non-sarcomatoid counterparts, and NF2 mutations were associated with sarcomatoid papillary RCCs. RELN mutations observed across all sRCC histologies were notable because RELN encodes Reelin, an extracellular matrix protease that regulates microtubule function and cell mobility ${ }^{101}$. Reelin inhibits TGF $\beta$-1-induced cell migration ${ }^{102}$, which might play a role in metastasis and tumour aggressiveness. However, overexpression of TGF $\beta$ - 1 can reciprocally suppress Reelin expression via the transcription factor Snail, a known activator of $\mathrm{EMT}^{102}$. Thus, in the event of Reelin loss (i.e. RELN mutation), upregulation of TGF $\beta$-1-induced cell migration and increased expression of mesenchymal markers are observed ${ }^{102}$. Wang et al. ${ }^{96}$ reported upregulation of TGF $\beta$ - 1 signalling across all sRCCs, which could be explained by similar elevation of RELN mutations across all histologies. This finding suggests that Reelin might be an anti-metastasis target for future drug development, although further investigation is necessary. Additionally, the study by Wang et al. ${ }^{96}$ was one of the few to report patient outcomes in relation to genomics and ccRCCs. Mutations in TP53, PTEN and RELN were associated with sarcomatoid change in ccRCCs $\left(P=1.64 \times 10^{-6}\right.$, $\mathrm{OR}=6.53$ ); however, in sarcomatoid ccRCCs, overall mutational frequencies of TP53, PTEN and RELN were lower than for $V H L(P=0.035)$, a hallmark of ccRCCs. These results suggest that mutations in TP53, PTEN and $R E L N$ occurred later in tumorigenesis than mutations in $V H L$. Notably, loss of $V H L$ or deletion of 3p21-25 genes (VHL, PBRM1, SETD2 and BAP1) was associated with increased overall survival, and mutations in TP53, $P T E N, R E L N$ were associated with decreased overall survival $^{96}$. Similarly, mutations in TP53, PTEN, RELN, $B A P 1$ and SETD2 were associated with sarcomatoid change, and mutations in VHL and PBRM1 negatively predicted sarcomatoid change.

In an unrelated molecular analysis of 62 aggressive primary uRCC specimens, sarcomatoid architectural patterns were found in a subset of uRCCs with NF2 loss. These results raise the possibility that aggressive uRCCs share a common molecular aetiology with sRCCs involving NF2 loss. Interestingly, NF2 is known to be a key effector of the Hippo-YAP pathway, which affects tissue growth, proliferation and differentiation 
Haematoxylin and eosin staining
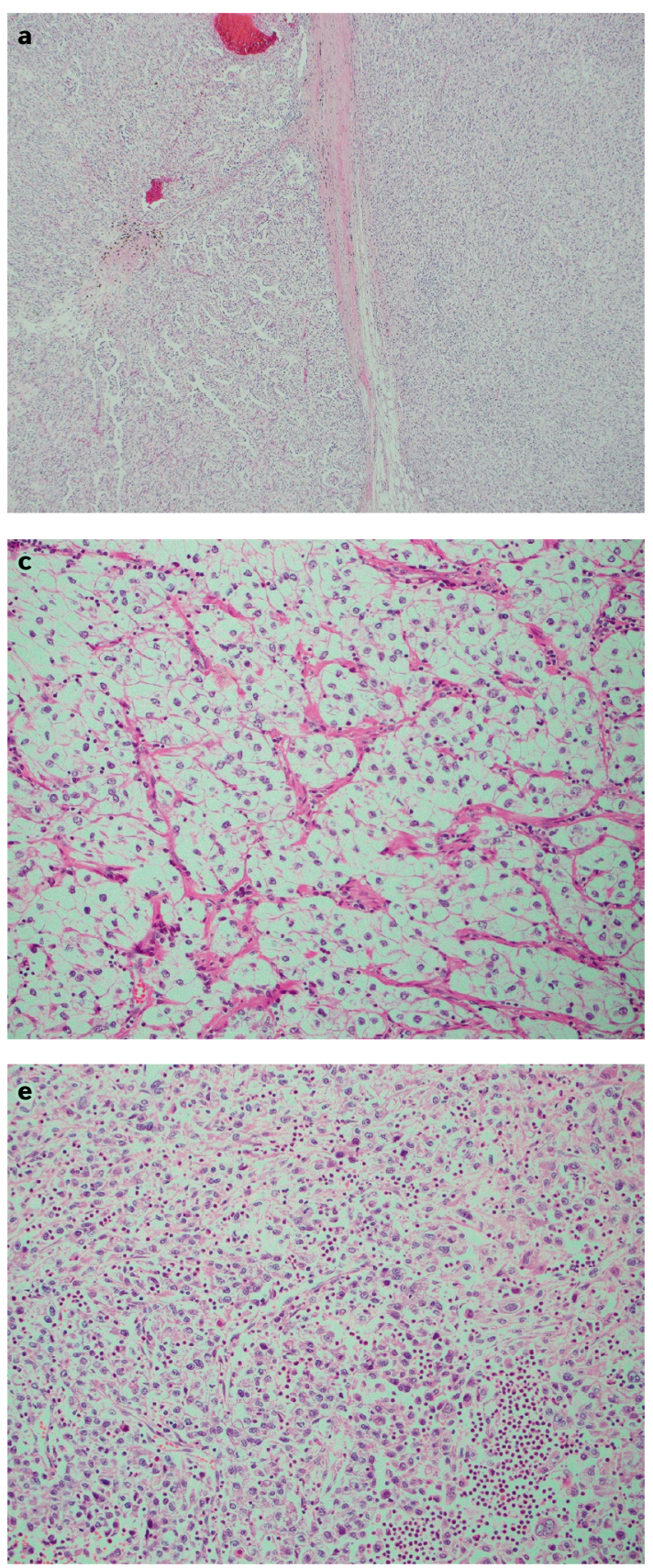

Immunostaining for PDL1
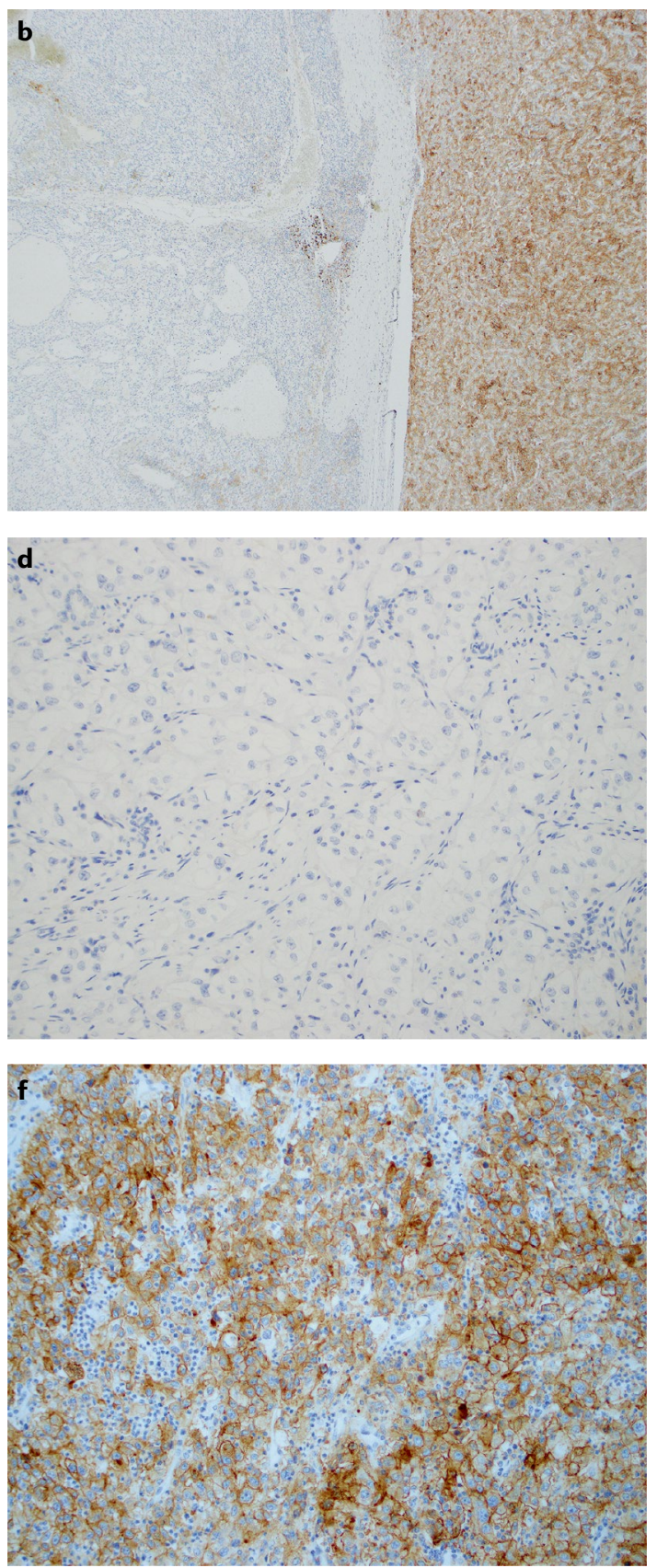

Fig. 4 | PDL1 expression in sRCC. Representative haematoxylin-and-eosin-stained images (left) of a 9p24.1-amplified clear cell renal cell carcinoma (ccRCC) with sarcomatoid transformation are depicted alongside corresponding immunostaining for PDL1 (right). Staining for PDL1 is absent in areas with a clear cell component and shows constitutive expression in areas with a sarcomatoid component, which is higher grade than the clear cell component. Constitutive expression of PDL1 in higher grade sarcomatoid components implies an underlying molecular event such as an amplification in JAK2, PDL1 and PDL2 at the 9p24.1 locus. Patients with this expression pattern have the potential for an enhanced response to immune checkpoint inhibitors owing to the increased expression of PDL1. Parts $\mathbf{a}$ and $\mathbf{b}$ show adjacent clear cell and sarcomatoid areas ( $\times 40$ magnification). Parts $\mathbf{c}$ and $\mathbf{d}$ show a representative area with clear cell morphology ( $\times 200$ magnification). Parts $\mathbf{e}$ and $\mathbf{f}$ show a representative area with sarcomatoid morphology ( $\times 200$ magnification). Reprinted from REF. ${ }^{99}$, Springer Nature Limited.

as well as cell migration ${ }^{103}$. Dysregulation of this pathway has been linked to uncoordinated cell growth and malignancy formation, and the key pathway effectors $Y A P$ and $T A Z$ often accumulate in the nucleus to drive proliferation ${ }^{104,105}$. Wild-type NF2 promotes a signalling cascade that results in phosphorylation of the transcription factors $Y A P$ and $T A Z$, resulting in inactivated cytoplasmic accumulation. However, mutated NF2 will deregulate this cascade, causing YAP and TAZ to remain unphosphorylated, with the ability to translocate into the 
cell nucleus and promote cell proliferation ${ }^{106}$. The postulated role of the Hippo-YAP pathway in sarcomatoid dedifferentiation is supported by the promotion of EMT by the key effectors of the pathway - YAP1 $\left(\mathrm{REFS}^{107-109}\right)$ and $T A Z^{110,111}$. YAP1 and TAZ are transcriptional regulators and are both associated with EMT signatures in solid tumours through upregulation of known EMT genes such as vimentin, fibronectin, SLUG and ZEB1 $1^{107}$.

In a 2020 study $^{112}$, Malouf and colleagues leveraged findings from previous sRCC genomic investigations that had shown mutations in Hippo-YAP pathway effectors, such as NF2 (REFS ${ }^{94,96,105}$ ) and FAT2 $\left(\right.$ REF. $\left.^{93}\right)$, to guide a dedicated study investigating Hippo-YAP pathway regulation in sRCCs. Using 49 clear cell sRCCs, targeted sequencing was performed in microdissected sarcomatoid and epithelial components and, together with non-microdissected sRCCs, compared with 268 non-sarcomatoid RCCs. The authors separately assessed the effects of NF2 knockout and reconstitution on sRCC proliferation both in vitro, using an NF2-mutant sRCC cell line, and in vivo, using male immunocompromised mouse xenografts. Results of targeted sequencing in 50 samples from 27 microdissected sRCC patient tumours showed mutations in VHL in $72 \%$, SETD2 in $40 \%, P B R M 1$ in $34 \%$ and $B A P 1$ in $26 \%{ }^{112}$. In the 22 non-microdissected sRCC cases, mutations were observed in VHL (68\%), and TP53 (27\%). Mutational burdens observed within the microdissected and non-microdissected sRCCs were in agreement with previously reported sRCC genomic evaluation studies ${ }^{93,94,96}$. Notably, in addition to the previously mentioned mutational findings, Hippo-YAP pathway mutational alterations (NF2, FAT1, LATS1, LATS2, YAP1 and TAZ) were observed in $20 \%$ of the 49 sRCC cases that underwent targeted sequencing. By contrast, $5 \%$ of the 268 non-sarcomatoid RCC patients had Hippo-YAP pathway mutations. Thus, the frequency of Hippo-YAP pathway mutations in sRCCs was significantly higher than in non-sarcomatoid RCCs $(P=0.001)$. Moreover, the authors then show that YAP1 knockout and NF2 reconstitution inhibit proliferation and invasion in an NF2-mutant sRCC cell line both in vitro and in vivo. In vitro, knockdown of Hippo-YAP effector YAP1 resulted in suppressed cell proliferation and invasion, as well as tumour growth, and induced morphological cellular change. In vivo, YAP1 suppression reduced tumour growth in NF2-mutant xenografts in male immunocompromised NOD-SCID IL2Rg-/- (NSG) mice. Overall, increased Hippo-YAP pathway alterations in sRCCs and the in vitro and in vivo observations of Malouf and colleagues suggest that interference with Hippo-YAP pathway function might disrupt sRCC tumour growth and could be a novel therapeutic target that warrants future study.

\section{Expression of immune checkpoint markers}

The rapid expansion of immunotherapies approved for RCCs has generated a desire to identify biomarkers indicative of treatment response, and PDL1 and programmed cell death 1 (PD1) expression have emerged as candidate biomarkers ${ }^{113,114}$. PDL1 is a cell surface protein that binds to PD1 on activated T-lymphocytes and decreases their anti-tumour activity ${ }^{115}$. Thus, tumour cells often upregulate PDL1 expression as a way of avoiding immune surveillance ${ }^{116,117}$. Tumour expression of PDL1 is associated with improved responses to PD1 and PDL1 blocking agents such as nivolumab or atezolizumab, compared with targeted agents such as sunitinib and everolimus ${ }^{113,118,119}$. In conventional RCCs, PDL1 is a poor prognostic marker that is associated with high-grade tumours and tumour necrosis ${ }^{120,121}$. The prognostic potential of PDL1 and PDL2 was explored in a study that immunohistochemically evaluated 425 RCC samples and correlated the expression of these proteins with patient clinicopathological features ${ }^{114}$. Overall, PDL1 expression was seen in $9.4 \%$ of samples and PDL2 in $49.6 \%{ }^{114}$. In ccRCCs, PDL1 expression was associated with adverse prognostic features, including higher WHO-ISUP grade, necrosis and sarcomatoid transformation (all $P<0.001)^{114}$. Furthermore, ccRCC PDL1 and PDL2 expression were both associated with shorter progression-free survival $(P<0.001$ and $P=0.033$, respectively) and shorter cancer-specific survival $(P<0.001 \text { and } P=0.010 \text {, respectively })^{114}$. In non-ccRCCs, PDL1 positivity was associated with higher tumour stage and grade when $10.9 \%$ of tumour cells and $56.4 \%$ of tumour infiltrating mononuclear cells were PDL1 positive ${ }^{121}$.

Expression of PD1 and PDL1 has been examined in sRCCs $^{122}$ (Supplementary Table 1). PD1 expression was observed in 25 of 26 sRCCs and PDL1 expression was observed in 14 tumours. By comparison, of 29 non-sarcomatoid ccRCC samples, 18 expressed PD1 and 5 expressed PDL1. Dual expression of PD1 and PDL1 was observed in 13 cases in the sRCC group compared with 1 in the non-sarcomatoid ccRCC group ${ }^{122}$. Similarly, in an immunohistochemical analysis of PDL1, PD1, CD4 and CD8 in 118 sRCC specimens and 92 non-sarcomatoid RCC (clear cell) specimens, sRCCs had higher PDL1 expression and higher PD1 ${ }^{+}$ $\mathrm{CD}^{+}$cell density than grade $4 \mathrm{ccRCCs}^{100}$. Moreover, $41 \%$ of sarcomatoid components within these tumours had an adaptive immune response phenotype (PDL1 ${ }^{+}$, tumour-infiltrating-lymphocyte $\left.{ }^{+}\right)^{100}$.

In a 2019 study $^{99}$, PDL1 expression was assessed in 398 high-grade RCCs, including 127 sRCCs, using $H$ scores (the product of the percentage of tumour cells with PDL1 expression and intensity of staining graded on a scale of $1-3$, maximum score $=300$ ). A large number of sRCCs $(\sim 27.6 \%)$ showed high PDL1 expression (H score $\geq 50$ ). Most of these cases exhibited adaptive patterns of PDL1 expression, but those with JAK2, PDL1 and PDL2 (9p24.1) amplifications showed constitutive patterns of PDL1 protein expression. Constitutive patterns of PDL1 expression seen in a subset of patients with sarcomatoid RCCs, in which every single tumour cell shows high levels of PDL1 expression, imply an underlying molecular event such as amplification of JAK2, PDL1 and PDL2 at the 9p24.1 locus. These patients may have enhanced or exceptional response to immunotherapy. However, analysis of outcomes based on PDL1 expression in this study did not reveal a significant prognostic effect, which was likely reflective of advanced disease. The WHO and International Society 
of Urologic Pathology (ISUP) classify sRCC as grade 4 disease and, accordingly, the majority of sRCC patients presented with advanced or high-stage disease ${ }^{99}$. Overall, these results add to an emerging body of data regarding the sRCC tumour immune microenvironment, which warrants further investigation into the possible benefits of PD1 and PDL1 immune checkpoint blockade therapy.

\section{Treatment}

\section{Surgical management}

In the setting of localized non-sarcomatoid RCCs, nephrectomy is a curative procedure. However, outcomes are less encouraging in patients with localized sRCCs $^{21,24}$. Approximately $77-80 \%$ of patients who receive nephrectomy with curative intent for localized sRCC recur within 5-26 months ${ }^{7,24}$. Bulky disease is usually present at initial presentation, commonly requiring radical nephrectomy for complete resection.

In addition to large primary tumours, approximately $60-80 \%$ of patients with an sRCC present with metastatic disease ${ }^{7,14,26}$. In these patients, cytoreductive nephrectomy can precede systemic treatment. In retrospective series investigating the surgical treatment of metastatic RCCs, cytoreductive nephrectomy before initiation of systemic therapy resulted in improved survival compared with systemic therapy alone ${ }^{123-127}$. In a 2014 study of 189 patients with sRCCs, median survival was 10.2 months in patients who underwent cytoreductive nephrectomy compared with 5.5 months in those who did not ${ }^{128}$. Indeed, in the largest epidemiological study of sRCCs to date, cytoreductive nephrectomy performed in patients with sRCC with good performance status had an observable survival benefit, albeit minor, compared with non-surgically treated patients ${ }^{14}$. In this study in 472 patients with metastatic sRCCs, the 1-, 3- and 5-year disease-specific survival for those who underwent nephrectomy was $33.7 \%, 10.8 \%$ and $6.2 \%$, respectively, compared with $11.5 \%, 1.9 \%$ and $0 \%$ in patients who did not. Overall, median disease-specific survival in those who underwent cytoreductive nephrectomy versus those who did not was 7 months (interquartile range (IQR) 3-17 months) versus 4 months (IQR 2-7 months) ${ }^{14}$. Moreover, multivariate cox proportional hazards modelling showed that cytoreductive nephrectomy was significantly and independently associated with improved disease-specific survival (HR 0.53, 95\% CI 0.43-0.66, $P<0.001$ (REF. ${ }^{14}$ ). Nevertheless, the role of cytoreductive nephrectomy in patients with sRCCs remains unclear, as retrospective data from existing reports are conflicting and no randomized controlled study exists to determine benefit. Although the aforementioned studies have shown data supporting the use of cytoreductive nephrectomy in sRCC patients, surgical management of metastatic sRCCs in the form of nephrectomy or metastasectomy has been alternatively argued to show variable benefit to survival and potentially delay initiation of systemic therapy owing to postoperative recovery ${ }^{129}$. In 419 patients who underwent cytoreductive nephrectomy, 62 patients with sRCCs had a median survival of 4.9 months compared with 17.7 months in those without sarcomatoid features ${ }^{15}$. Furthermore, contrary to non-sarcomatoid RCCs, those with sRCC were shown to have no survival benefit from post-nephrectomy metastasectomy ${ }^{130}$. A major difficulty of this debate is that, given the low rates of sRCC detection on preoperative imaging and biopsy ${ }^{15,19,37}$, most patients are not known to have an sRCC until after the nephrectomy is performed. Nevertheless, cytoreductive nephrectomy in selected patients with sRCCs might have palliative benefits, such as decreasing local symptoms of bulky disease and gross haematuria ${ }^{131}$.

\section{Radiotherapy}

RCC is largely considered a radio-resistant tumour ${ }^{132}$. The use of conventional radiotherapy as a primary treatment modality for RCCs was initially hindered by preclinical evidence suggesting inherent radio-resistance ${ }^{133}$ and implementation was further impeded by an absence of demonstrable benefit in clinical studies ${ }^{134,135}$. Accordingly, radiotherapy has been used sparingly in the treatment of RCCs for the past 50 years $^{136}$. Similarly, sRCC responses to radiotherapy have been underwhelming. In a study in 408 patients with non-metastatic sRCCs, overall or disease-specific survival at 1,3 and 5 years in those who received adjuvant radiotherapy was not significantly different from those who underwent surgical treatment alone ${ }^{137}$. Currently, radiotherapy is indicated mainly as a palliative measure for patients with metastatic disease or recurrent local tumour growth ${ }^{132,136}$. In the palliative setting, metastatic burden, metastasis accessibility, resectability and patient performance status are important harm-benefit management considerations ${ }^{138}$. Radiotherapy provides patients with a localized non-invasive therapy option that can alleviate pain, decrease neurological symptoms and improve haematuria from symptomatic metastases in otherwise non-surgical candidates ${ }^{132,139}$. Work is ongoing to assess the role of stereotactic ablative radiotherapy in RCCs, which delivers a higher ( $\geq 8 \mathrm{~Gy}$ ) dose of radiation to the tumour than conventional radiotherapy $(\leq 2 \mathrm{~Gy})^{140,141}$. Data on new forms of radioablative therapy for the treatment of primary RCCs, such as proton beam therapy, are limited. A 2017 case report about a patient with an inoperable RCC, owing to morbid obesity and multiple comorbidities, is the only published instance of proton therapy for a primary RCC. Although a decline in glomerular filtration rate was observed from $34 \mathrm{ml} / \mathrm{min} / 1.73 \mathrm{~m}^{2}$ to $29 \mathrm{ml} / \mathrm{min} / 1.73 \mathrm{~m}^{2}$, no clinical symptoms of late radiation-induced toxic effects were observed and the patient had remained asymptomatic by the last follow-up point of 1 year ${ }^{142}$. This is a hypothesis-generating report, but it remains a single-institution case report and further research is required to determine whether proton therapy is appropriate in both RCCs and sRCCs. To date, no study has assessed the outcomes of patients with sRCCs treated exclusively with radiotherapy, and the available findings indicate that outcomes would presumably not be promising.

\section{Systemic therapy}

Systemic treatment for sRCC has been predominantly ineffective with few therapies producing durable responses $^{35,143,144}$. Combinations of cytotoxic therapies, 
targeted therapies and immunotherapies are being used with varying effects. Selection of chemotherapeutic agents was initially driven in part by the reported benefits of doxorubicin and ifosfamide in sarcomas ${ }^{145}$. In 1987, Sella et al. ${ }^{36}$ performed one of the earliest studies assessing systemic therapy in patients with sRCCs. The study reports outcomes for 44 patients after doxorubicin chemotherapy, non-doxorubicin chemotherapy, hormonal therapy (medroxyprogesterone 17-acetate and androgen therapy), and interferon- $\alpha$ as separate groups. Overall median survival was 6-12 months. Notably, two patients had complete responses with doxorubicin and 4 patients treated with interferon- $\alpha$ had a median survival of 41 months $^{36}$. These findings spurred future studies to focus on chemotherapy and immunotherapy as potential treatment options moving forward.

Chemotherapy. Chemotherapy efficacy in sRCCs (TABLE 1) has been explored over the past two decades in four notable studies ${ }^{146-149}$. First, a retrospective analysis of 8 patients with sRCCs treated with doxorubicin-based therapy and observed a median survival of 20-60 months in 3 patients ${ }^{146}$. Second, a multi-institutional phase II trial in 23 patients with sRCCs of combination doxorubicin and ifosfamide reported no clinical responses: median time to progression was 2.2 months and overall median survival was $<4$ months $^{147}$. Third, a study in 10 patients with sRCC receiving combination antimetabolite gemcitabine and topoisomerase inhibitor doxorubicin observed two complete responses and one partial response $\mathrm{i}^{148}$. The 2 patients who had complete responses remained disease-free at 6 years and 8 years ${ }^{150}$. Finally, a prospective phase II clinical trial evaluated the efficacy of combination doxorubicin and gemcitabine in patients with previously untreated sRCCs ${ }^{149}$. The Eastern Cooperative Oncology Group (ECOG) 8802 trial reported responses to treatment in $6(16 \%)$ patients (5 partial responses and 1 complete response), and stable disease in $10(26 \%)$ patients. Median overall survival

Table 1 | Chemotherapy for the treatment of sRCCs

\begin{tabular}{|c|c|c|c|c|}
\hline Study & Drugs & $n$ & $\begin{array}{l}\text { Response rate } \\
\text { (number of patients) }\end{array}$ & Outcomes \\
\hline $\begin{array}{l}\text { Culine et al. } \\
(1995)^{146}\end{array}$ & Doxorubicin & 14 & OR $37.5 \%$ (3) & $\begin{array}{l}\text { Median OS } \\
20-60 \text { months }\end{array}$ \\
\hline $\begin{array}{l}\text { Escudier } \\
\text { et al. } \\
(2002)^{147}\end{array}$ & $\begin{array}{l}\text { Doxorubicin + } \\
\text { ifosfamide }\end{array}$ & 23 & $\begin{array}{l}\text { OR 0\% (0) } \\
\text { SD 26.1\% (6) } \\
\text { PD 17.4\% (4) }\end{array}$ & $\begin{array}{l}\text { Median TTP } \\
2.2 \text { months; } \\
\text { median OS } \\
3.9 \text { months }\end{array}$ \\
\hline $\begin{array}{l}\text { Nanus et al. } \\
(2004)^{148}\end{array}$ & $\begin{array}{l}\text { Doxorubicin+ } \\
\text { gemcitabine }\end{array}$ & 18 (10 sRCCs) & $\begin{array}{l}\text { CR 20\% (2) } \\
\text { SD 20\% (2) } \\
\text { PR 10\% (1) } \\
\text { MR 10\% (1) }\end{array}$ & $\begin{array}{l}\text { Median } \\
\text { duration of } \\
\text { response } \\
\text { (entire } \\
\text { cohort)= } \\
5 \text { months }\end{array}$ \\
\hline $\begin{array}{l}\text { Haas et al. } \\
(2012)^{149}\end{array}$ & $\begin{array}{l}\text { Doxorubicin + } \\
\text { gemcitabine }\end{array}$ & 38 & $\begin{array}{l}\text { CR 3\% (1) } \\
\text { PR 13\% (5) } \\
\text { SD 26\% (10) } \\
\text { PD 35\% (13) }\end{array}$ & $\begin{array}{l}\text { Median OS } \\
8.8 \text { months; } \\
\text { median PFS= } \\
3.5 \text { months }\end{array}$ \\
\hline
\end{tabular}

$\mathrm{CR}$, complete response; $\mathrm{MR}$, mixed response; OR, objective response; OS, overall survival; PR, partial response; SD, stable disease; sRCCs, sarcomatoid renal cell carcinomas; TTP, time to treatment progression; PFS, progression free survival.
(OS) was 8.8 months, and median progression-free survival (PFS) was 3.5 months ${ }^{149}$. Treatment response based on percentage sarcomatoid from available data (19 tumours from 19 patients) showed 7 tumours with 0-49\% sarcomatoid features, 1 tumour with $50-74 \%$ sarcomatoid features and 11 tumours with $75-100 \%$ sarcomatoid features. The data on progression, stable disease, partial response and complete response from the ECOG 8802 trial, although descriptive and not subject to formal statistics, suggest that patients whose tumours had a high percentage $(>75 \%)$ of sarcomatoid component might benefit from cytotoxic therapy, irrespective of the underlying subtype $\mathrm{e}^{149}$.

Overall, the use of cytotoxic chemotherapy in sRCCs has yielded poor outcomes. Overall survival has never reached $>9$ months and progression of disease has occurred within $<4$ months in $17.4-35 \%$ of patients (TABLE 1). Moreover, phase II sRCC chemotherapy trials resulted in PFS of 2.2-3.5 months, and OS of 3.9-8.8 months ${ }^{147,149}$. Overall, cytotoxic agents have failed to prove an effective therapy option for patients with sRCCs.

Targeted therapy. During the era of targeted therapies (2006-2015) (TABLE 2) the number of studies exploring the effectiveness of these interventions in sRCC increased. One study examined the efficacy of the VEGF-inhibitor sorafenib in 15 patients who had progressed on combination doxorubicin and gemcitabine ${ }^{151}$. Before receiving sorafenib, no responses had been observed on combination chemotherapy and median time to progression was 6.6 months. Patients who received subsequent sorafenib had a mean time to progression of 10.9 months ${ }^{151}$. In a retrospective study assessing 43 patients with sRCCs treated with VEGFtargeted agents (sunitinib, sorafenib, or bevacizumab), a $19 \%$ partial response rate and a median PFS and OS of 5.3 months and 11.8 months, respectively, was observed $^{143}$. Six patients with ccRCC histology and $<20 \%$ sarcomatoid dedifferentiation in their tumour achieved partial responses ${ }^{143}$. In a larger retrospective series, VEGF inhibitor therapy (sunitinib, sorafenib, axitinib, pazopanib, tivozanib or bevacizumab) in 230 patients with metastatic sRCCs compared with 2,056 patients with non-sarcomatoid RCCs was explored ${ }^{152}$. Over $93 \%$ of patients received anti-VEGF agents as first-line therapy and objective responses were slightly less frequent in sRCCs than in non-sarcomatoid RCC (20\% vs 26\%). Median PFS and OS were 4.5 months and 10.4 months in sRCCs and 7.8 months and 22.5 months in non-sarcomatoid RCCs, respectively ${ }^{152}$. Another retrospective analysis of 23 patients with sarcomatoid ccRCCs treated with mTOR inhibitors (temsirolimus or everolimus) reported a $13 \%$ partial response rate and $30 \%$ stable disease rate ${ }^{144}$. Median PFS was 3.5 months and median OS was 8.2 months. None of the patients experienced a complete response and overall the authors deemed patient response to therapy to be poor ${ }^{144}$. A separate study examined survival in patients with sRCCs who underwent nephrectomy and were treated with systemic therapy in the cytokine era (1987-2005) and the targeted therapy era (2006-2015) ${ }^{19}$. The cytokine 
Table 2 | Targeted therapy for treatment of sRCC

\begin{tabular}{|c|c|c|c|c|}
\hline Study & Drugs & $n$ & Response rate & Outcomes \\
\hline $\begin{array}{l}\text { Golshayan } \\
\text { et al. }(2009)^{143}\end{array}$ & $\begin{array}{l}\text { Sunitinib }+ \text { sorafenib }+ \\
\text { bevacizumab }\end{array}$ & 43 & $\begin{array}{l}\text { PR } 19 \%(8)^{\mathrm{a}} ; \mathrm{SD} \\
49 \%(21) ; \mathrm{PD} \\
33 \%(14)\end{array}$ & $\begin{array}{l}\text { Median PFS } 5.3 \text { months; } \\
\text { median OS } 11.8 \text { months }\end{array}$ \\
\hline $\begin{array}{l}\text { Staehler et al. } \\
(2010)^{151}\end{array}$ & $\begin{array}{l}\text { Sorafenib in patients with } \\
\text { sRCC who had progressed on } \\
\text { doxorubicin + gemcitabine }\end{array}$ & 9 & $\begin{array}{l}\text { PR } 11.1 \%(1) ; \text { SD } \\
44.4 \%(4)\end{array}$ & Mean TTP 10.9 months \\
\hline $\begin{array}{l}\text { Jonasch et al. } \\
(2011)^{156}\end{array}$ & $\begin{array}{l}\text { Gemcitabine + capecitabine } \\
\text { + bevacizumab }\end{array}$ & $\begin{array}{l}28 \mathrm{mRCCs} \\
(10 \mathrm{sRCCs})\end{array}$ & Not reported & $\begin{array}{l}\text { Median PFS (sRCC) } 3.9 \text { months; } \\
\text { median OS (sRCC) } 9.0 \text { months; } \\
\text { median PFS (nsRCC) } \\
6.1 \text { months; median OS } \\
\text { (nsRCC) } 10.9 \text { months }\end{array}$ \\
\hline $\begin{array}{l}\text { Voss et al. } \\
(2014)^{144}\end{array}$ & Temsirolimus or everolimus & $\begin{array}{l}85 \mathrm{mRCC} \\
\text { (23 sRCCs) }\end{array}$ & $\begin{array}{l}\text { PR (sRCC) } 13 \% \\
\text { (3); SD (sRCC) } \\
30 \% \text { (7); PD } \\
\text { (sRCC) } 57 \% \text { (13) }\end{array}$ & $\begin{array}{l}\text { Median PFS (sRCC) } 3.5 \text { months; } \\
\text { median OS (sRCC) } 8.2 \text { months }\end{array}$ \\
\hline $\begin{array}{l}\text { Kyriakopoulous } \\
\text { et al. }(2015)^{152}\end{array}$ & $\begin{array}{l}\text { Targeted therapies } \\
\text { (sunitinib, sorafenib, axitinib, } \\
\text { bevacizumab, temsirolimus, } \\
\text { pazopanib, everolimus or } \\
\text { tivozanib) }\end{array}$ & $\begin{array}{l}2,286 \text { mRCCs } \\
\text { (230 sRCCs) }\end{array}$ & $\begin{array}{l}\text { OR 20\% (46); PR } \\
18 \% \text { (17); CR 3\% } \\
\text { (3); SD 36\% (34); } \\
\text { PD 43\% (40) }\end{array}$ & $\begin{array}{l}\text { Median PFS (sRCC) } 4.5 \text { months; } \\
\text { median OS (sRCC) } 10.4 \\
\text { months; median PFS (nsRCC) } \\
7.8 \text { months; median OS } \\
\text { (nsRCC) } 22.5 \text { months }\end{array}$ \\
\hline $\begin{array}{l}\text { Keskin et al. } \\
(2017)^{19}\end{array}$ & $\begin{array}{l}\text { Cytokine (1987-2005) } \\
\text { and targeted therapy } \\
(2006-2015) \text { eras }\end{array}$ & $\begin{array}{l}122 \text { cytokine, } \\
77 \text { targeted } \\
\text { therapy }\end{array}$ & Not reported & $\begin{array}{l}\text { 1-year OS benefit observed } \\
\text { in patients treated in the } \\
\text { targeted therapy era; median } \\
\text { OS } 16.5 \text { months }\end{array}$ \\
\hline
\end{tabular}

era cohort, which assessed IL-2, IFNa and chemotherapeutic systemic agents, comprised 122 patients and the targeted therapy era cohort, which assessed sunitinib, sorafenib, bevacizumab, axitinib, pazopanib, erlotinib, nivolumab, ipilimumab, temsirolimus or everolimus systemic agents, comprised 77 patients. The authors noted a 12-month OS benefit in patients with sRCC treated in the targeted therapy era $(P=0.011)$. However, this improvement in OS was not durable and disappeared at 3-5 years to become indistinguishable from that of patients treated in the cytokine era. Notably, the OS improvement occurred in patients at intermediate risk but not in patients with poor-risk disease ${ }^{19}$. Rationale for this finding is likely linked to the aggressive and treatment-resistant nature of sRCCs and perhaps further compounded by the added poor prognosis of poorrisk features. Indeed, at the time of this study, poor-risk patients had not experienced an improvement in survival in $\sim 20$ years, a finding in agreement with similar reports ${ }^{14,19}$. The authors concluded that survival in patients with sRCCs was not substantially augmented by the addition of either IL-2 or IFNa, or targeted therapy ${ }^{19}$. A similar study reviewed outcomes after systemic therapy in 63 patients with metastatic sRCCs, stratifying by first-line agent: anti-angiogenesis-targeted therapy (sunitinib, sorafenib, temsirolimus or everolimus; $n=34$ ), cytokine therapy (IFNa, IL-2, or combination; $n=20$ ) and chemotherapy (geldanamycin, cetuximab, bortezomib or gemcitabine; $n=9)^{153}$. Of 63 patients, the retrospective analysis found that 5 patients had an objective response ( 1 cytokine, 4 sunitinib), and the overall cohort median PFS and OS were 3 months and
10 months, respectively. Median PFS was 4.4 months in sunitinib-treated patients versus 2 months for all other patients $(P=0.03)^{153}$.

Targeted therapy studies have reported overall partial response rates of $11.1-19 \%$, with progression of disease observed in $33-57 \%$ of patients within 1 year (TABLE 2). Rapid recurrences and poor response rates have curtailed enthusiasm for the use of targeted therapies in sRCCs, as studies have shown little to modest improvement in sRCC survival outcomes.

Combination therapy. The effectiveness of combination chemotherapy plus targeted therapy has been explored in clinical trials in patients with sRCCs ${ }^{154,155}$ (TABLE 3). A phase II trial evaluated combination gemcitabine plus sunitinib in sRCCs and poor-risk RCCs ${ }^{154}$. In 39 patients with sRCCs, objective response rate (ORR) was $26 \%$, with a median time to progression of 5 months and a median OS of 10 months. Patients whose tumours had $>10 \%$ sarcomatoid dedifferentiation had greater clinical benefit than patients with $\leq 10 \%$ sarcomatoid dedifferentiation $(P=0.04)$. A retrospective study analysed combination gemcitabine, capecitabine and bevacizumab response in 10 patients with metastatic $\mathrm{RCC}^{156}$. PFS and OS in patients with sRCCs was 3.9 months and 9 months, respectively, compared with 6.1 months and 10.9 months in patients with non-sarcomatoid RCCs ${ }^{156}$. In addition, a phase II trial of capecitabine, gemcitabine and bevacizumab in 34 patients with metastatic sRCCs was conducted to explore the use of combination chemotherapy with targeted therapy agents ${ }^{155}$. Overall objective response was $20 \%$ (5 partial, 1 complete), and the 
Table 3 | Combination chemotherapy with targeted-therapy or immunotherapy for treatment of sRCC

\begin{tabular}{|c|c|c|c|c|}
\hline Study & Drugs & $n$ & Response rate & Outcomes \\
\hline \multirow{2}{*}{$\begin{array}{l}\text { Escudier et al. } \\
(2002)^{147}\end{array}$} & \multirow{2}{*}{$\begin{array}{l}\text { Doxorubicin }+ \\
\text { ifosfamide }\end{array}$} & \multirow[t]{2}{*}{23} & \multirow[t]{2}{*}{ OR $0 \%$} & Median PFS 2.2 months \\
\hline & & & & Median OS 3.9 months \\
\hline \multirow{3}{*}{$\begin{array}{l}\text { Haas, et al. } \\
(2012)^{149}\end{array}$} & \multirow{3}{*}{$\begin{array}{l}\text { Doxorubicin + } \\
\text { gemcitabine }\end{array}$} & \multirow[t]{3}{*}{39} & PR $12.8 \%(5)$ & Median PFS 3.5 months \\
\hline & & & CR $2.6 \%(1)$ & Median OS 8.8 months \\
\hline & & & SD $26 \%(10)$ & \\
\hline \multirow{4}{*}{$\begin{array}{l}\text { Michaelson, et al. } \\
(2015)^{154}\end{array}$} & \multirow{4}{*}{$\begin{array}{l}\text { Sunitinib + } \\
\text { gemcitabine }\end{array}$} & \multirow{4}{*}{$\begin{array}{l}72 \text { RCCs, } \\
\text { (39 sRCCs) }\end{array}$} & OR (sRCC) $26 \%(10)$ & Median TTP (sRCC) 5 months \\
\hline & & & PR (sRCC) 23\% (9) & Median OS (sRCC) 10 months \\
\hline & & & CR (sRCC) $3 \%(1)$ & \\
\hline & & & $\mathrm{SD}(\mathrm{sRCC}) 38 \%(15)$ & \\
\hline \multirow[t]{4}{*}{$\begin{array}{l}\text { Haas et al. } \\
(2016)^{157}\end{array}$} & \multirow[t]{4}{*}{$\begin{array}{l}\text { Sunitinib } \pm \\
\text { gemcitabine }\end{array}$} & \multirow{4}{*}{$\begin{array}{l}\text { 71: } 35 \text { sunitinib + } \\
\text { gemcitabine, } \\
36 \text { sunitinib) }\end{array}$} & $\begin{array}{l}\text { OR (sunitinib }+ \\
\text { gemcitabine) } 20 \% \text { (7) }\end{array}$ & $\begin{array}{l}\text { Median PFS (sunitinib + gemcitabine) } \\
23 \text { weeks }\end{array}$ \\
\hline & & & OR (sunitinib) $11.1 \%$ (4) & Median PFS (sunitinib) 13 weeks \\
\hline & & & & $\begin{array}{l}\text { Median OS (sunitinib + gemcitabine) } \\
41 \text { weeks }\end{array}$ \\
\hline & & & & Median OS (sunitinib) 33 weeks \\
\hline \multirow{4}{*}{$\begin{array}{l}\text { McDermott et al. } \\
(2016)^{171}\end{array}$} & \multirow[t]{4}{*}{ Atezolizumab } & \multirow{4}{*}{$\begin{array}{l}70 \mathrm{mRCCs} \\
\text { (18 sRCCs) }\end{array}$} & OR (sRCC) $22 \%(4)$ & Median PFS (sRCC) 4.2 months \\
\hline & & & & Median OS (sRCC) 26.2 months \\
\hline & & & & 1-year OS rate (sRCC) 93\% \\
\hline & & & & 2-year OS rate (sRCC) 57\% \\
\hline \multirow{3}{*}{$\begin{array}{l}\text { Maiti, et al. } \\
(2017)^{155}\end{array}$} & \multirow{3}{*}{$\begin{array}{l}\text { Capecitabine + } \\
\text { gemcitabine }+ \\
\text { bevacizumab }\end{array}$} & \multirow[t]{3}{*}{$34^{\mathrm{a}}$} & OR $16.6 \%(5)$ & Median PFS 5.5 months \\
\hline & & & CR $3.3 \%(1)$ & Median TTP 4.2 months \\
\hline & & & PR $13.3 \%(4)$ & Median OS 12 months \\
\hline
\end{tabular}

$\mathrm{CR}$, complete response; $\mathrm{mRCCs}$, metastatic renal cell carcinomas; OS, overall survival; OR, objective response; PFS, progressionfree survival; PR, partial response; RCC, renal cell carcinomas; SD, stable disease; sRCCs, sarcomatoid renal cell carcinomas; TTP, time to progression. ${ }^{\circ}$ Four patients were excluded from response analysis, and one patient was excluded from survival analysis.

disease control rate was $73 \%$. Median time to treatment failure was 4.2 months, median PFS was 5.5 months and median OS was 12 months ${ }^{155}$. Preliminary results of the phase II ECOG 1808 trial of sunitinib with or without gemcitabine in patients with sRCCs were presented at the 2016 American Society of Clinical Oncology annual meeting ${ }^{157}$. Of 87 patients, 47 were randomly assigned to sunitinib plus gemcitabine and 40 to sunitinib only. Response rates for assessable patients were 20\% (7/35) for the sunitinib plus gemcitabine arm and $11.1 \%(4 / 36)$ for the sunitinib arm. Median PFS and OS were 5.29 months and 9.43 months for sunitinib plus gemcitabine, and 2.99 months and 7.59 months for sunitinib ${ }^{157}$.

Combination therapy for sRCCs has yielded objective, partial and complete response rates ranging from $0 \%$ to $26 \%, 12.8 \%$ to $23 \%$, and $2.6 \%$ to $3.3 \%$, respectively. When quantitatively assessing the available data on combination therapy, response rates in sRCCs remain low even when combining two effective conventional RCC therapies.

High-dose IL-2. The rapid development of immunotherapies has added a new dimension of potential systemic therapy options for patients with sRCCs. A 2017 study reported outcomes of 21 patients with metastatic sRCCs who received high-dose IL-2 following nephrectomy ${ }^{158}$. Overall response rate was $10 \%$, and $5 \%$ of patients experienced complete response. Localized disease was associated with improved responses to high-dose IL-2, and median PFS and OS were 7.9 months and 30.5 months, respectively ${ }^{158}$. The authors conclude that high-dose IL-2 offers modest overall response rates similar to responses seen at the time in other immunotherapy studies, which had complete response ranges of $6-9.3 \%$ and partial responses of $8.3-18 \%^{35,159-161}$. However, since this publication, high-dose IL-2 therapy in RCCs has fallen out of favour and has been largely replaced with newer immune checkpoint blockade agents owing to the limited overall response rates, high rate of treatment-related deaths $(\sim 4-6 \%)^{161}$ and poor tolerability ${ }^{162,163}$ in the form of high rates of grade 3 and grade 4 adverse events ${ }^{164,165}$ associated with IL-2 therapy.

Immune checkpoint blockade therapy. Immune checkpoint inhibitors have seen the most progress compared with other systemic therapies for sRCCs. Tumours with either constitutive or high levels of adaptive PDL1 expression patterns and high levels of tumourinfiltrating lymphocytes (TILs) are the most likely to respond to immune checkpoint blockade ${ }^{166}$. Previous studies indicate that sRCCs have higher expression of PDL1 on tumour cells and PD1 on TILs than nonsarcomatoid RCCs ${ }^{122,167}$. Furthermore, concomitant expression is reported in up to $50 \%$ of sRCCs of any subtype, compared with $3 \%$ of non-sarcomatoid RCCs ${ }^{122}$, suggesting that the PD1-PDL1 axis has an active role in sRCC. Additionally, in a study of 118 sRCCs, $40 \%$ of sarcomatoid components harboured both PDL1 
expression and TILs, compared with $8 \%$ of the corresponding epithelial components and only $1 \%$ of the nonsarcomatoid RCC control group ${ }^{100}$. These results suggest a pattern of immune resistance in sRCCs, making investigations into anti-PDL1 and PD1 agents a sensible choice for therapeutic exploitation.

Taken together, four case reports have documented a rapid response to PD1 inhibition by nivolumab in patients with sRCCs. A 2017 report involved a patient with clinically localized chromophobe sRCC initially treated with nephrectomy who, 7 years later, developed local disease recurrence ${ }^{168}$. The patient was treated with adjuvant combination doxorubicin and ifosfamide with minimal effect and underwent palliative debulking surgery followed by six cycles of second-line nivolumab therapy, which resulted in a partial response ${ }^{168}$. A 2015 report described a patient with a metastatic papillary sRCC with rapid disease progression on carboplatin-gemcitabine, sunitinib and gemcitabine given in sequence ${ }^{169}$. At 3 weeks following a single dose of nivolumab, clinical, biological and radiological responses were observed ${ }^{169}$. Similarly, in a 2018 report, a complete response to the PD1 checkpoint inhibitor nivolumab was reported in a 67-year-old man with clear cell sRCC who initially experienced disease progression after a 4-month treatment of combination sunitinib plus gemcitabine ${ }^{170}$. At 1 month after starting nivolumab the patient experienced partial resolution of metastatic sites and at 6 months of treatment PET-CT showed complete resolution of all known metastatic disease. Nivolumab was continued for 2 years and PET-CT at that time showed no evidence of recurrence ${ }^{170}$. In a 2019 report, 2 patients with sRCCs with documented amplifications of $P D L 1$ at $9 \mathrm{p} 24.1$ were reported to have a dramatic response to immunotherapy ${ }^{99}$. The first patient received pembrolizumab as a second-line therapy. Pretreatment imaging demonstrated diffuse metastatic disease, and on-treatment imaging showed stable disease at 16 months. The second patient received atezolizumab as a first-line therapy. Imaging prior to immunotherapy documented metastases to the lungs, liver, bone, thoracic adenopathy and nephrectomy bed. At 3 months post-treatment, imaging showed a decrease in the size and number of pulmonary metastases, adenopathy, and nephrectomy bed involvement, with radiological evidence of stable disease at 14 months. A combination of PDL1 amplification, coupled with $J A K 2$-activation-dependent upregulation of PDL1 and adaptive and/or induced expression leads to extremely high levels of PDL1 expression in tumours. These patients might, therefore, show an enhanced response to immune checkpoint blockade therapy.

Prospective immune checkpoint blockade studies and sRCCs. Five prospective studies have investigated different forms of immune checkpoint blockade therapy in sRCCs, which continues to show the strongest improvement in outcomes compared with any previous systemic therapy for this disease. Most of the trial results have now been published in abstract form (TABLE 4), with completed publications anticipated.

The safety and clinical activity of anti-PDL1 atezolizumab in metastatic RCCs were evaluated in a phase Ia trial that included patients with sarcomatoid features ${ }^{171}$. Patients with WHO/ISUP grade 4 RCC and/or sarcomatoid features $(n=18)$ were separately assessed for ORR, PFS and OS. ORR was 33\% for patients with sarcomatoid dedifferentiation and $25 \%$ for patients with grade 4 disease. Median PFS and OS for patients with grade 4 RCC and/or sarcomatoid features was 4.2 months and 26.2 months, respectively ${ }^{171}$. Median PFS and OS was 5.6 months and 28.9 months for the entire cohort, respectively ${ }^{171}$.

A post hoc exploratory analysis of the CheckMate214 trial $^{172}$, which originally assessed the anti-CTLA4 agent ipilimumab plus the anti-PDL1 agent nivolumab compared with single-agent anti-VEGF sunitinib in patients with intermediate-risk or poor-risk RCC, identified patients with sarcomatoid histology and measured efficacy and safety outcomes in this subpopulation ${ }^{173}$. Of 139 patients with sRCC (74 receiving ipilimumab plus nivolumab and 65 receiving sunitinib), the median follow-up duration was 47.7 months and ORR after ipilimumab plus nivolumab or sunitinib administration was $60.8 \%$ vs $23.1 \%$ compared with $41.9 \%$ vs $29.4 \%$ in the remaining participants, respectively. Overall PDL1 expression was higher in patients with sRCCs than in other patients (51\% vs $27.5 \%$ ), and patients with sRCCs with $\geq 1 \%$ PDL1 expression treated with ipilimumab plus nivolumab had a longer median OS than those treated with sunitinib ${ }^{173}$. Overall, patients with sRCCs treated with ipilimumab plus nivolumab had improved median PFS (26.5 vs 5.1 months, $P=0.0093$ ), complete response rates $(18.9 \%$ vs $3.1 \%)$, partial response rates (41.9\% vs $20.0 \%$ ) and median OS (not reached vs 14.2 months, $P=0.0155$ ) compared with those treated with sunitinib ${ }^{173,174}$. The 42 -month overall survival probability was $50.1 \%$ (95\% CI 37.9-61.2) with

Table 4 | Prospective data assessing response of sRCC to immune checkpoint blockade

\begin{tabular}{|c|c|c|c|c|c|c|c|c|c|}
\hline Clinical trial & Phase & $N$ & ORR & PR & CR & Median PFS & Median OS & Intervention arm (n) & $\begin{array}{l}\text { Standard of } \\
\text { care arm (n) }\end{array}$ \\
\hline CheckMate-214 ${ }^{173}$ & III & 139 & $60.8 \%$ & $41.9 \%$ & $18.9 \%$ & 26.5 months & NR & Ipilimumab + nivolumab (74) & Sunitinib (65) \\
\hline Keynote- $426^{177}$ & III & 105 & $58.80 \%$ & $47 \%$ & $11.8 \%$ & NR & NR & Pembrolizumab + axitinib (51) & Sunitinib (54) \\
\hline Javelin Renal-101 ${ }^{180}$ & III & 108 & $46.8 \%$ & $42.6 \%$ & $4.3 \%$ & 7.0 months & - & Avelumab + axitinib (47) & Sunitinib (61) \\
\hline IMmotion151 ${ }^{181}$ & III & 142 & $49 \%$ & $39 \%$ & $10 \%$ & 8.3 months & 21.7 months & Atezolizumab + Bevacizumab (68) & Sunitinib (74) \\
\hline
\end{tabular}

-, not specified; CR, complete response; NR, not reached; OS, overall survival; ORR, objective response rate; PFS, progression-free survival; PR, partial response.

ancludes sRCCs and grade 4 RCCs. ORR, PFS and OS values presented in this table are based on results from the intervention arm. 
ipilimumab plus nivolumab versus $22.6 \%$ (95\% CI 13.3 33.4) with sunitinib. Based on these results, the Society for Immunotherapy of Cancer recommended ipilimumab plus nivolumab combination immunotherapy as a first-line treatment option for patients with sRCCs ${ }^{175}$.

The KEYNOTE-426 trial $^{176}$ assessed anti-PD1 pembrolizumab plus anti-VEGF axitinib as a first-line therapy for patients with metastatic RCCs. In 2019, abstracted outcomes for a subgroup of 105 patients with sRCCs were presented ${ }^{177}$. The PDL1 combined positive score $\geq 1$ (calculated by dividing the number of PDL1-positive tumour cells, macrophages and lymphocytes by the total number of cells and multiplying the result by 100) for patients with sRCCs was $74.5-79.6 \%$. Of 105 patients with sRCCs, 51 were randomly assigned to receive pembrolizumab plus axitinib and 54 to sunitinib. ORR was $58.8 \%$ versus $31.5 \%$ and the complete response rate was $11.8 \%$ versus $0 \%$ in the pembrolizumab plus axitinib versus sunitinib arms, respectively. Percentage of tumour shrinkage was greater for pembrolizumab plus axitinib than for sunitinib: $80 \%$ vs $50 \%$ of patients experienced $a \geq 30 \%$ decrease change from baseline in target lesions according to RECIST v1.1 (REF. ${ }^{178}$ ) criteria, respectively. Furthermore, PFS at 12 months was $57 \%$ in the pembrolizumab plus axitinib arm compared with $26 \%$ in the sunitinib arm, respectively. Overall, the PFS HR was 0.54 (95\% CI 0.29-1.00) and the OS HR was 0.58 (95\% CI 0.21-1.59) for pembrolizumab plus axitinib. The authors concluded that pembrolizumab plus axitinib has substantial activity in sRCCs $^{177}$.

Abstracted post hoc data regarding sRCC outcomes from the phase III JAVELIN-Renal 101 trial $^{179}$ were presented at the 2019 annual European Society of Medical Oncology in Barcelona, Spain. This trial originally assessed the anti-PDL1 checkpoint inhibitor avelumab plus axitinib versus sunitinib in previously untreated patients with advanced RCCs. The primary end points were PFS and OS in patients with PDL1-positive tumours. Of 886 patients enrolled in the trial, 442 were assigned to receive avelumab plus axitinib and 444 to receive sunitinib. Among the 560 patients with PDL1-positive tumours, median PFS was 13.8 months with avelumab plus axitinib, compared with 7.2 months with sunitinib (disease progression or death HR 0.61, 95\% CI 0.47-0.79; $P<0.001$ ). ORR in patients with PDL1-positive tumours was $55.2 \%$ with avelumab plus axitinib and $25.5 \%$ with sunitinib. Overall, PFS was longer in the avelumab plus axitinib arm as a first-line agent in PDL1-positive tumours. In the post hoc sRCC analysis ${ }^{179}, 108$ patients with sRCCs were identified. Of these patients, 47 were randomly assigned avelumab plus axitinib, whereas 61 received sunitinib. The avelumab plus axitinib arm achieved an improvement in ORR (46.8\%, 95\% CI 32.1-61.9; OR 3.249 95\% CI $1.300-8.236)$ versus sunitinib $(21.3 \%$, 95\% CI 11.9-33.7), and 2 patients receiving avelumab plus axitinib had a complete response compared with none receiving sunitinib. Additionally, treatment with combination agents resulted in a 3-month improvement in PFS (HR 0.57, 95\% CI 0.325-1.003), as well as a 2.4-month improvement in duration of response for patients with
sRCC when compared with sunitinib ${ }^{180}$. Overall, these results suggest that sRCCs may benefit from an immunotherapy plus VEGF-targeted therapy combination. Continued reporting beyond 24 months is highly anticipated and will further add to the growing body of data on the use and efficacy of immune checkpoint blockade in patients with sRCCs.

A pre-specified subgroup analysis ${ }^{181}$ of patients with sRCC was conducted based on the phase III randomized IMmotion 151 trial ${ }^{182}$, which was designed to assess atezolizumab plus bevacizumab versus sunitinib in previously untreated patients with advanced or metastatic RCC. The original trial results showed prolonged PFS for patients receiving atezolizumab plus bevacizumab within the intent-to-treat population (11.2 months vs 7.7 months, HR $0.74,95 \%$ CI $0.57-0.96, P=0.0217)$. The subgroup analysis assessed the effectiveness of atezolizumab plus bevacizumab versus sunitinib in patients with sRCCs. Of 142 patients with sRCC, 68 received atezolizumab plus bevacizumab and 74 received sunitinib. Patients with sRCC who received atezolizumab plus bevacizumab had longer PFS (8.3 months vs 5.3 months, HR 0.52, 95\% CI 0.34-0.79) and higher ORR (49\% vs $14 \%)$ than those who received sunitinib. Median overall survival in the sRCC atezolizumab plus bevacizumab group versus sunitinib group was 21.7 months versus 15.4 months, respectively. Notably, more patients with sRCCs who received atezolizumab plus bevacizumab achieved complete response (10\% vs $3 \%$ ). Results from this subgroup analysis suggest improved clinical efficacy with atezolizumab plus bevacizumab compared with sunitinib for patients with sRCCs, as shown by improved PFS, OS and ORR.

In summary, immune checkpoint inhibitors are a promising therapeutic strategy in the treatment of sRCCs. The available data support a higher expression of PDL1 in sRCC tumour cells and improved outcomes after immune checkpoint blockade. Indeed, immune checkpoint inhibitors have shown increased ORR compared with sunitinib ( $50 \%$ vs $20 \%$, RR 2.15 , $P<0.00001$ ) overall, with $\sim 40 \%$ decrease in progression (HR 0.56, $P<0.0001$ ) and overall mortality risk (HR $0.56, P=0.001)^{183}$. As new data become available, the PD1-PDL1 signalling axis will likely be an important therapeutic target to explore in the future.

Ongoing clinical trials. Currently, one clinical trial ${ }^{184}$ is evaluating systemic therapy applications specifically for patients with sRCC (Supplementary Table 2), although recruitment for this trial is currently suspended because of the COVID-19 pandemic. This single-arm, phase I trial is designed to assess the feasibility and safety of combination avelumab and gemcitabine, for use in patients with metastatic sRCCs. As a phase I clinical trial, the primary outcome for this study is the number of adverse events experienced by patients. Previous reports indicate that sRCCs have higher expression of PDL1 on tumour cells than non-sarcomatoid RCCs ${ }^{122}$, making avelumab a practical choice for trial investigation. Gemcitabine has previously been paired with a number of systemic agents - either other chemotherapies or targeted therapies, such as gemcitabine-doxorubicin, 
gemcitabine-sunitinib, and gemcitabine-capecitabinebevacizumab - with varying and generally limited success $^{148,149,151,154-156,169}$. However, gemcitabine in combination with an anti-PDL1 agent has not previously been explored in sRCCs.

Notably, a currently recruiting phase III trial ${ }^{185}$ will assess first-line therapy with the anti-PDL1 agent nivolumab combined with ipilimumab. Standard treatment of nivolumab and ipilimumab followed by nivolumab alone will be compared with cabozantinib and nivolumab in patients with untreated metastatic RCCs. Patients with metastatic disease who have not received systemic therapy for RCCs are eligible for inclusion: the primary outcome is overall survival, and the secondary outcomes include PFS, objective response and complete response. Although not specific to sRCC, this trial will include patients with metastatic sRCCs. At the conclusion of this trial, a sub-analysis of patients with metastatic sRCCs would help to confirm or refute current speculation. Specifically, studies have reported sRCCs to have higher expression of PD1 and PDL1 $\left(\right.$ REFS $\left.^{99,122,168,169,174}\right)$ and a high infiltration of regulatory $\mathrm{T}\left(\mathrm{T}_{\text {reg }}\right)$ cells, which constitutively express CTLA4; expression of both is associated with poor prognosis in RCC, indicating an immuno-exhaustive state ${ }^{186-189}$. Thus, treatment of patients with sRCCs with a combination of an anti-PDL1 agent and an anti-CTLA4 agent seems potentially promising. No trial to date has completed enrolment and specifically assessed this combination in sRCCs and, therefore, the data generated from this study are highly anticipated to help to address this speculation.

\section{Management strategies in sRCCs}

An sRCC is an aggressive variant of an RCC, and most patients present with large primary tumours and synchronous metastases. Unfortunately, regardless of treatment choice, 5-year survival for patients with sRCCs has remained steady at $23.5-33 \%$ for the past three decades $^{14,23,26}$ despite improved surgical techniques and systemic therapies. Currently, no reliable method of identifying sRCC preoperatively exists. Owing to the heterogeneous distribution of sarcomatoid regions within an sRCC tumour, successful identification with biopsy only occurs in $\sim 7.5 \%$ of cases ${ }^{19}$. Indeed, although localized sRCC has worse outcomes than localized non-sarcomatoid RCC, durable responses have been shown following nephrectomy in the localized setting ${ }^{24}$. Thus, for localized disease, surgical resection remains the standard of care ${ }^{24}$.

However, the majority of sRCC patients present with advanced or metastatic disease for which the role of nephrectomy is controversial. A 2009 study based on cytokine systemic therapy era data previously suggested that patients with metastatic sRCCs first receive a trial of systemic therapy and are only offered cytoreductive nephrectomy if a response was demonstrated ${ }^{15}$. A larger 2019 study advocated that cytoreductive nephrectomy in good performance status patients ${ }^{14}$ is appropriate. Taking an individualized patient-centred approach when deciding whether or not to surgically intervene in sRCC patients is imperative and caution is recommended.
To date, a search for an effective systemic therapy for sRCC is still ongoing. Cytotoxic and targeted therapies have both shown poor outcomes as single agents or in combinations. However, the immunotherapy era has seen a renaissance of new drugs that show encouraging levels of response in sRCCs. Multiple contemporary studies show that sRCCs have increased co-expression of PD1 on tumour-infiltrating lymphocytes and PDL1 on sRCC tumour cells compared with conventional subtypes of RCC ${ }^{99,100,122}$, which suggests that immune checkpoint blockade therapies might be of increased benefit. Post hoc analyses from clinical trials have shown increased benefit of immunotherapy in combination with targeted therapy agents such as anti-VEGF therapy, and have achieved all-time high objective response rates in advanced or metastatic disease of $55.2-58.8 \%{ }^{177,179}$. Furthermore, anti-CTLA4 systemic agents such as ipilimumab might be beneficial against sRCC tumours in conjunction with anti-PD1 and/or PDL1 therapy ${ }^{190}$ : results from a post hoc analysis of the CheckMate214 trial ${ }^{172}$ show an objective response of $56.7 \%$ vs $19.2 \%$, and a complete response in $18.3 \%$ vs $0 \%$ of patients after ipilimumab plus nivolumab versus sunitinib, respectively ${ }^{174}$. Contemporary data have shown increased infiltration of $\mathrm{T}_{\text {reg }}$ cells, which constitutively express CTLA4 (REF. ${ }^{191}$ ), into sRCCs compared with conventional RCCs, providing further evidence of the potential benefits of combination immunotherapy treatment.

The growing number and availability of immune checkpoint inhibitors such as anti-CTLA4, anti-PD1 and anti-PDL1 systemic agents in the setting of compelling data supporting higher expression of PD1 and PDL1 and greater infiltration of $\mathrm{T}_{\text {reg }}$ cells in sRCCs than in non-sarcomatoid RCCs suggests that this signalling axis could be a critical therapeutic target. Future clinical trials should focus on therapies such as anti-CTLA4, anti-PDl, anti-PDL1 and combinations with targeted therapy agents that have been shown to augment the cytotoxic tumour immune microenvironment $99,122,168,169,173,186-189$ with the aim of improving progression and survival outcomes in patients with sRCCs.

Although findings from ongoing and future studies are awaited, current data from immune checkpoint inhibitor trials suggest that treatment for patients with metastatic RCCs and confirmed sRCCs on renal biopsy should begin with immune checkpoint inhibitor combination therapy. Indeed, improved PFS and ORR with combination therapy have been observed in subgroup analyses of sRCC patients within the randomized controlled IMmotion151 trial assessing atezolizumab plus bevacizumab versus sunitinib in advanced or metastatic RCCs ${ }^{181}$ The use of cytoreductive nephrectomy in metastatic patients with sRCCs should be considered, though with a high threshold for surgical eligibility, as conflicting data on survival benefit are available, with most recent population-based data suggesting a possible benefit in good-risk group patients.

\section{Conclusions}

The paucity of data on sRCCs highlights the need for continued research into the biology, diagnostics and effective treatment options for patients with this disease, 
as responses to conventional therapies have been underwhelming and leave few options for those diagnosed with sRCC. Cytoreductive nephrectomy for patients with sRCC with metastatic disease is controversial, but literature as recent as 2019 (REF. ${ }^{14}$ ) advocates for resection at any stage in patients with good performance status. Reports of higher expression of PD1 and PDL1 in sRCCs than in non-sarcomatoid RCCs has generated growing interest in immune checkpoint blockade therapies in combination with other systemic agents, and at least two clinical trials are underway to directly explore this relationship. Ultimately, large collaborative efforts are needed to improve our understanding of sRCCs in order to devise more effective treatments for this lethal disease.

Published online 13 October 2020
1. Siegel, R. L., Miller, K. D. \& Jemal, A. Cancer statistics, 2019. CA Cancer J. Clin. 69, 7-34 (2019).

Capitanio, U. et al. Epidemiology of renal cell carcinoma. Eur. Urol. 75, 74-84 (2019).

3. Bray, F. et al. Global cancer statistics 2018 GLOBOCAN estimates of incidence and mortality worldwide for 36 cancers in 185 countries. CA Cancer J. Clin. 68, 394-424 (2018).

4. Motzer, R. J. et al. Kidney cancer, version 2.2017, NCCN clinical practice guidelines in oncology. J. Natl Compr. Canc. Netw. 15, 804-834 (2017).

5. Delahunt, B. Sarcomatoid renal carcinoma: the final common dedifferentiation pathway of renal epithelial malignancies. Pathology 31, 185-190 (1999).

6. de Peralta-Venturina, M. et al. Sarcomatoid differentiation in renal cell carcinoma: a study of 10 cases. Am. J. Surg. Pathol. 25, 275-284 (2001).

7. Mian, B. M. et al. Prognostic factors and survival of patients with sarcomatoid renal cell carcinoma. J. Urol. 167, 65-70 (2002).

8. Shuch, B. et al. Quality of pathological reporting for renal cell cancer: implications for systemic therapy, prognostication and surveillance. BJU Int. 108 343-348 (2011).

9. Shuch, B. et al. Histologic evaluation of metastases in renal cell carcinoma with sarcomatoid transformation and its implications for systemic therapy. Cancer 116, 616-624 (2010)

10. Cheville, J. C. et al. Sarcomatoid renal cell carcinoma: an examination of underlying histologic subtype and an analysis of associations with patient outcome. Am. J. Surg. Pathol. 28, 435-441 (2004).

11. Kim, T. et al. Using percentage of sarcomatoid differentiation as a prognostic factor in renal cell carcinoma. Clin. Genitourin. Cancer 13, 225-230 (2015).

12. Brookman-May, S. et al. Prognostic effect of sarcomatoid dedifferentiation in patients with surgically treated renal cell carcinoma: a matched-pair analysis. Clin. Genitourin. Cancer 11, 465-470 (2013).

13. $\mathrm{Gu}, \mathrm{L}$. et al. Presence of sarcomatoid differentiation as a prognostic indicator for survival in surgically treated metastatic renal cell carcinoma. J. Cancer Res. Clin. Oncol. 143, 499-508 (2017).

14. Alevizakos, M., Gaitanidis, A., Nasioudis, D., Msaouel, P. \& Appleman, L. J. Sarcomatoid renal cell carcinoma: population-based study of 879 patients. Clin. Genitourin Cancer 17 e447-e453 (2019).

15. Shuch, B. et al. Cytoreductive nephrectomy for kidney cancer with sarcomatoid histology-is up-front resection indicated and, if not, is it avoidable? J. Urol. 182, 2164-2171 (2009).

16. Korenbaum, C. et al. Treatments, outcomes, and validity of prognostic scores in patients with sarcomatoid renal cell carcinoma: a 20-year singleinstitution experience. Clin. Genitourin. Cancer 16, e577-e586 (2018)

17. Cangiano, T. et al. Sarcomatoid renal cell carcinoma: biologic behavior, prognosis, and response to combined surgical resection and immunotherapy. J. Clin. Oncol. 17, 523-528 (1999)

18. Ro, J. Y., Ayala, A. G., Sella, A., Samuels, M. L. \& Swanson, D. A. Sarcomatoid renal cell carcinoma: clinicopathologic. A study of 42 cases. Cancer 59 , 516-526 (1987)

19. Keskin, S. K. et al. Outcomes of patients with renal cell carcinoma and sarcomatoid dedifferentiation treated with nephrectomy and systemic therapies: comparison between the cytokine and targeted therapy eras. J. Urol. 198, 530-537 (2017).

20. Lucca, I., Klatte, T., Fajkovic, H., de Martino, M. \& Shariat, S. F Gender differences in incidence and outcomes of urothelial and kidney cancer. Nat. Rev. Urol. 12, 585-592 (2015).

21. Zhang, B. Y. et al. A novel prognostic model for patients with sarcomatoid renal cell carcinoma. BJU Int. 115, 405-411 (2015).
22. Adibi, M. et al. Percentage of sarcomatoid component as a prognostic indicator for survival in renal cell carcinoma with sarcomatoid dedifferentiation. Urol. Oncol. 33, 427.e417-423 (2015).

23. Trudeau, V. et al. Comparison of oncologic outcomes between sarcomatoid and clear cell renal cell carcinoma. World J. Urol. 34, 1429-1436 (2016).

24. Merrill, M. M. et al. Clinically nonmetastatic renal cell carcinoma with sarcomatoid dedifferentiation: natural history and outcomes after surgical resection with curative intent. Urol. Oncol. 33, 166.e121-169 (2015).

25. Russo, P. et al. Survival rates after resection for localized kidney cancer: 1989 to 2004. Cancer 113, 84-96 (2008)

26. Shuch, B. et al. Impact of pathological tumour characteristics in patients with sarcomatoid renal cell carcinoma. BJU Int. 109, 1600-1606 (2012).

27. Weisel, W., Dockerty, M. B. \& Priestley, J. T. Sarcoma of the kidney. J. Urol. 50, 564-573 (1943).

28. Lee-Tsün, H. \& Willis, R. A. Renal carcino-sarcoma, true and false. J. Pathol. Bacteriol. 85, 139-144 (1963).

29. Farrow, G. M., Harrison, E. G. Jr \& Utz, D. C. Sarcomas and sarcomatoid and mixed malignant tumors of the kidney in adults. 3. Cancer 22 556-563 (1968).

30. Thoenes, W., Storkel, S. \& Rumpelt, H. J. Histopathology and classification of renal cell tumors (adenomas, oncocytomas and carcinomas). The basic cytological and histopathological elements and their use for diagnostics. Pathol. Res. Pract. 181, 125-143 (1986).

31. Storkel, S. et al. Classification of renal cell carcinoma: Workgroup No. 1. Union Internationale Contre le Cancer (UICC) and the American Joint Committee on Cancer (AJCC). Cancer 80, 987-989 (1997).

32. Kovacs, G et al. The Heidelberg classification of renal cell tumours. J. Pathol. 183, 131-133 (1997).

33. Delahunt, B. et al. The International Society of Urological Pathology (ISUP) grading system for renal cell carcinoma and other prognostic parameters. Am. J. Surg. Pathol. 37, 1490-1504 (2013).

34. Moch, H., Cubilla, A. L., Humphrey, P. A., Reuter, V. E. $\&$ Ulbright, T. M. The 2016 WHO classification of tumours of the urinary system and male genital organs - part A: renal, penile, and testicular tumours. Eur. Urol. 70, 93-105 (2016).

35. Shuch, B., Bratslavsky, G., Linehan, W. M. \& Srinivasan, R. Sarcomatoid renal cell carcinoma: a comprehensive review of the biology and current treatment strategies. Oncologist 17, 46-54 (2012).

36. Sella, A., Logothetis, C. J., Ro, J. Y., Swanson, D. A. \& Samuels, M. L. Sarcomatoid renal cell carcinoma. A treatable entity. Cancer 60, 1313-1318 (1987).

37. Abel, E. J. et al. Limitations of preoperative biopsy in patients with metastatic renal cell carcinoma: comparison to surgical pathology in 405 cases. BJU Int. 110, 1742-1746 (2012).

38. Schieda, N. et al. Diagnosis of sarcomatoid renal cell carcinoma with CT: evaluation by qualitative imaging features and texture analysis. AJR Am. J. Roentgenol 204, 1013-1023 (2015).

39. Rosenkrantz, A. B., Chandarana, H. \& Melamed, J. MRI findings of sarcomatoid renal cell carcinoma in nine cases. Clin. Imaging 35, 459-464 (2011).

40. Takeuchi, M. et al. Characteristic MRI findings of sarcomatoid renal cell carcinoma dedifferentiated from clear cell renal carcinoma: radiologicalpathological correlation. Clin. Imaging 37, 908-912 (2013).

41. Takeuchi, M. et al. MRI for differentiation of renal cell carcinoma with sarcomatoid component from other renal tumor types. Abdom. Imaging 40, 112-119 (2015).

42. Jeong, D. et al. Quantification of sarcomatoid differentiation in renal cell carcinoma on magnetic resonance imaging. Quant. Imaging Med. Surg. 8, 373-382 (2018).

43. Fuser, D., Hedberg, M. L., Dehner, L. P., Dehdashti, F. \& Siegel, B. A. Extensive metastatic Sarcomatoid renal cell carcinoma evaluated by (18)F-FDG PET/CT: a case report and review of literature. J. Kidney Cancer VHL 5, 1-6 (2018)

44. Thambugala, G. M., Mohamed, A., O'Neill, G. F. \& Fulham, M. J. Sarcomatoid renal cell carcinoma: rapid dissemination detected on FDG PET-CT Australas. Radiol. 50, 604-606 (2006)

45. Hyodo, T. et al. Widespread metastases from sarcomatoid renal cell carcinoma detected by 18F-FDG positron emission tomography/computed tomography. Japn. J. Radiol. 27, 111-114 (2009)

46. Nadebaum, D. P., Hofman, M. S., Mitchell, C. A., Siva, S. \& Hicks, R. J. Oligometastatic renal cell carcinoma with sarcomatoid differentiation demonstrating variable imaging phenotypes on 68Ga-PSMA and 18F-FDG PET/CT: a case report and review of the literature. Clin. Genitourin. Cancer 16 , 1-5 (2018).

47. Vogel, C. et al. Imaging in suspected renal-cell carcinoma: systematic review. Clin. Genitourin. Cancer 17, e345-e355 (2019).

48. Donat, S. M. et al. Follow-up for clinically localized renal neoplasms: AUA guideline. J. Urol. 190, 407-416 (2013)

49. Escudier, B. et al. Renal cell carcinoma: ESMO clinical practice guidelines for diagnosis, treatment and follow-up. Ann. Oncol. 25, iii49-iii56 (2014).

50. Ljungberg, B. et al. in European Association of Urology Guidelines. 2018 Edition. Vol. presented at the EAU Annual Congress Copenhagen 2018 (European Association of Urology Guidelines Office, 2018).

51. Kuroda, N., Toi, M., Hiroi, M. \& Enzan, H. Review of sarcomatoid renal cell carcinoma with focus on clinical and pathobiological aspects. Histol. Histopathol. 18 , 551-555 (2003).

52. Reuter, V. E. Sarcomatoid lesions of the urogenital tract. Semin. Diagn. Pathol. 10, 188-201 (1993).

53. Kwak, C. et al. Sarcomatoid differentiation as a prognostic factor for immunotherapy in metastatic renal cell carcinoma. J. Surg. Oncol. 95, 317-323 (2007).

54. Leibovich, B. C. et al. Histological subtype is an independent predictor of outcome for patients with renal cell carcinoma. J. Urol. 183, 1309-1315 (2010).

55. Casuscelli, J. et al. Chromophobe renal cell carcinoma: results from a large single-institution series. Clin. Genitourin. Cancer 17, 373-379.e374 (2019).

56. Akhtar, M., Tulbah, A., Kardar, A. H. \& Ali, M. A. Sarcomatoid renal cell carcinoma: the chromophobe connection. Am. J. Surg. Pathol. 21, 1188-1195 (1997)

57. Kuroda, N et al. Acquired cystic disease-associated renal cell carcinoma with sarcomatoid change and rhabdoid features. Ann. Diagn. Pathol. 15, 462-466 (2011).

58. Chen, Y. B. et al. Hereditary leiomyomatosis and renal cell carcinoma syndrome-associated renal cancer: recognition of the syndrome by pathologic features and the utility of detecting aberrant succination by immunohistochemistry. Am. J. Surg. Pathol. 38, 627-637 (2014).

59. Udager, A. M. et al. Hereditary leiomyomatosis and renal cell carcinoma (HLRCC): a rapid autopsy report of metastatic renal cell carcinoma. Am. J. Surg. Pathol. 38, 567-577 (2014).

60. He, H. \& Magi-Galluzzi, C. Epithelial-to-mesenchymal transition in renal neoplasms. Adv. Anat. Pathol. 21, 174-180 (2014).

61. Tong, G. X. et al. Expression of PAX8 in normal and neoplastic renal tissues: an immunohistochemical study. Mod. Pathol. 22, 1218-1227 (2009). 
62. Ozcan, A., de la Roza, G., Ro, J. Y., Shen, S. S. \& Truong, L. D. PAX2 and PAX8 expression in primary and metastatic renal tumors: a comprehensive comparison. Arch. Pathol. Lab. Med. 136, 1541-1551 (2012)

63. Tickoo, S. K. et al. Immunohistochemical expression of hypoxia inducible factor-1 alpha and its downstream molecules in sarcomatoid renal cell carcinoma. J. Urol. 177, 1258-1263 (2007).

64. Kutikov, A. et al. Renal mass biopsy: always, sometimes, or never? Eur. Urol. 70, 403-406 (2016).

65. Auger, M. et al. Fine-needle aspiration cytology of sarcomatoid renal cell carcinoma: a morphologic and immunocytochemical study of 15 cases. Diagn. Cytopathol. 9, 46-51 (1993).

66. Abel, E. J. et al. Multi-quadrant biopsy technique improves diagnostic ability in large heterogeneous renal masses. J. Urol. 194, 886-891 (2015).

67. Manley, B. J. \& Hsieh, J. J. Sarcomatoid renal cell carcinoma: genomic insights from sequencing of matched sarcomatous and carcinomatous components. Transl. Cancer Res. 5, S160-s165 (2016).

68. Conant, J. L., Peng, Z., Evans, M. F., Naud, S. \& Cooper, K. Sarcomatoid renal cell carcinoma is an example of epithelial-mesenchymal transition. J. Clin. Pathol. 64, 1088-1092 (2011).

69. Jones, T. D. et al. Clonal divergence and genetic heterogeneity in clear cell renal cell carcinomas with sarcomatoid transformation. Cancer 104, 1195-1203 (2005)

70. Wick, M. R. \& Swanson, P. E. Carcinosarcomas: current perspectives and an historical review of nosological concepts. Semin. Diagn. Pathol. 10 118-127 (1993)

71. Kalluri, R. EMT: when epithelial cells decide to become mesenchymal-like cells. J. Clin. Invest. 119, 1417-1419 (2009)

72. Zeisberg, M. \& Neilson, E. G. Biomarkers for epithelial-mesenchymal transitions. J. Clin. Invest. 119, 1429-1437 (2009).

73. Iwatsuki, M. et al. Epithelial-mesenchymal transition in cancer development and its clinical significance. Cancer Sci. 101, 293-299 (2010).

74. De Craene, B. \& Berx, G. Regulatory networks defining EMT during cancer initiation and progression. Nat. Rev. Cancer 13, 97-110 (2013).

75. Huber, M. A., Kraut, N. \& Beug, H. Molecular requirements for epithelial-mesenchymal transition during tumor progression. Curr. Opin. Cell Biol. 17, 548-558 (2005).

76. Cano, A. et al. The transcription factor snail controls epithelial-mesenchymal transitions by repressing E-cadherin expression. Nat. Cell Biol. 2, 76-83 (2000).

77. Guarino, M., Rubino, B. \& Ballabio, G. The role of epithelial-mesenchymal transition in cancer pathology. Pathology 39, 305-318 (2007).

78. Lamouille, S., Xu, J. \& Derynck, R. Molecular mechanisms of epithelial-mesenchymal transition Nat. Rev. Mol. Cell Biol. 15, 178-196 (2014).

79. Piloto, S. \& Schilling, T. F. Ovo 1 links Wnt signaling with $\mathrm{N}$-cadherin localization during neural crest migration. Development 137, 1981-1990 (2010).

80. Gumbiner, B. M. Regulation of cadherin adhesive activity. J. Cell Biol. 148, 399-404 (2000).

81. Sircar, K. et al. Biphasic components of sarcomatoid clear cell renal cell carcinomas are molecularly similar to each other, but distinct from, non-sarcomatoid renal carcinomas. J. Pathol. 1, 212-224 (2015).

82. Hsieh, C. H. et al. Co-existence of epithelioid and fibroblastoid subsets in a sarcomatoid renal carcinoma cell line revealed by clonal studies. Anticancer. Res. 33, 4875-4889 (2013).

83. Lopez-Lago, M. A. et al. Genomic deregulation during metastasis of renal cell carcinoma implements a myofibroblast-like program of gene expression. Cancer Res. 70, 9682-9692 (2010).

84. Braga, E. A., Fridman, M. V., Loginov, V. I., Dmitriev, A. A. \& Morozov, S. G. Molecular mechanisms in clear cell renal cell carcinoma: role of miRNAs and hypermethylated miRNA genes in crucial oncogenic pathways and processes. Front. Genet. 10, 320 (2019).

85. Mann, J. et al. Regulation of myofibroblast transdifferentiation by DNA methylation and MeCP2: implications for wound healing and fibrogenesis. Cell Death Differ. 14, 275-285 (2007).

86. Dumont, N. et al. Sustained induction of epithelial to mesenchymal transition activates DNA methylation of genes silenced in basal-like breast cancers. Proc. Natl Acad. Sci. USA 105, 14867-14872 (2008).
87. Xu, M. et al. miR-203 inhibition of renal cancer cell proliferation, migration and invasion by targeting of FGF2. Diagn. Pathol. 10, 24 (2015)

88. Schneider, M., Hansen, J. L. ¿ Sheikh, S. P. S100A4: a common mediator of epithelial-mesenchymal transition, fibrosis and regeneration in diseases? J. Mol. Med. 86, 507-522 (2008).

89. Helfman, D. M. Kim, E. J., Lukanidin, E. \& Grigorian, M. The metastasis associated protein S100A4: role in tumour progression and metastasis. Br. J. Cancer 92, 1955-1958 (2005).

90. Oda, H., Nakatsuru, Y. \& Ishikawa, T. Mutations of the $\mathrm{p} 53$ gene and $\mathrm{p} 53$ protein overexpression are associated with sarcomatoid transformation in renal cell carcinomas. Cancer Res. 55, 658-662 (1995).

91. Linehan, W. M., Lerman, M. I. \& Zbar, B. Identification of the von Hippel-Lindau (VHL) gene. Its role in renal cancer. JAMA 273, 564-570 (1995)

92. Clark, P. E. The role of VHL in clear-cell renal cell carcinoma and its relation to targeted therapy. Kidney Int. 76, 939-945 (2009).

93. $\mathrm{Bi}, \mathrm{M}$. et al. Genomic characterization of sarcomatoid transformation in clear cell renal cell carcinoma Proc. Natl Acad. Sci. USA 113, 2170-2175 (2016).

94. Malouf, G. G. et al. Genomic characterization of renal cell carcinoma with sarcomatoid dedifferentiation pinpoints recurrent genomic alterations. Eur. Urol. 70 348-357 (2016)

95. Wei, S. \& Al-Saleem, T. The pathology and molecular genetics of sarcomatoid renal cell carcinoma: a mini-review. J. Kidney Cancer VHL 4, 19-23 (2017).

96. Wang, Z. et al. Sarcomatoid renal cell carcinoma has a distinct molecular pathogenesis, driver mutation profile, and transcriptional landscape. Clin. Cancer Res. 23, 6686-6696 (2017).

97. Bergerot, P., Agarwal, N., Pal, S. K. \& Jones, J. Sarcomatoid renal cell carcinoma: the apple doesn't fall far from the tree. Clin. Cancer Res. 23, 6381-6383 (2017)

98. Creighton, C. J. et al. Comprehensive molecular characterization of clear cell renal cell carcinoma. Nature 499, 43-49 (2013).

99. Gupta, S. et al. JAK2/PD-L1/PD-L2 (9p24.1) amplifications in renal cell carcinomas with sarcomatoid transformation: implications for clinical management. Mod. Pathol. https://doi.org/10.1038/ s41379-019-0269-x (2019).

100. Kawakami, F. et al. Programmed cell death ligand 1 and tumor-infiltrating lymphocyte status in patients with renal cell carcinoma and sarcomatoid dedifferentiation. Cancer 123, 4823-4831 (2017).

101. Wasser, C. R. \& Herz, J. Reelin: neurodevelopmental architect and homeostatic regulator of excitatory synapses. J. Biol. Chem. 292, 1330-1338 (2017).

102. Yuan, Y., Chen, H., Ma, G., Cao, X. \& Liu, Z. Reelin is involved in transforming growth factor-beta 1 -induced cell migration in esophageal carcinoma cells. PLoS One 7, e31802 (2012)

103. Kulkarni, A., Chang, M. T., Vissers, J. H. A., Dey, A. \& Harvey, K. F. The Hippo pathway as a driver of select human cancers. Trends Cancer 6, 781-796 (2020).

104. Meng, Z., Moroishi, T. \& Guan, K. L. Mechanisms of Hippo pathway regulation. Genes Dev. 30, 1-17 (2016).

105. Chen, Y. B. et al. Molecular analysis of aggressive renal cell carcinoma with unclassified histology reveals distinct subsets. Nat. Commun. 7, 13131 (2016).

106. Zhao, B., Tumaneng K. \& Guan, K. L. The Hippo pathway in organ size control, tissue regeneration and stem cell self-renewal. Nat. Cell Biol. 13, 877-883 (2011).

107. Shao, Diane D. et al. KRAS and YAP1 converge to regulate EMT and tumor survival. Cell 158, 171-184 (2014).

108. Lamar, J. M. et al. The Hippo pathway target, YAP, promotes metastasis through its TEAD-interaction domain. Proc. Natl Acad. Sci. USA 109, E2441-E2450 (2012).

109. Liu, Y. et al. YAP modulates TGF- $\beta 1$-induced simultaneous apoptosis and EMT through upregulation of the EGF receptor. Sci. Rep. 7, 45523 (2017).

110. Li, Z. et al. The Hippo transducer TAZ promotes epithelial to mesenchymal transition and cancer stem cell maintenance in oral cancer. Mol. Oncol. 9 1091-1105 (2015)

111. Wang, Y., Liu, J., Ying, X., Lin, P. C. \& Zhou, B. P. Twist-mediated epithelial-mesenchymal transition promotes breast tumor cell invasion via inhibition of hippo pathway. Sci. Rep. 6, 24606 (2016).

112. Malouf, G. G. et al. Molecular characterization of sarcomatoid clear cell renal cell carcinoma unveils new candidate oncogenic drivers. Sci. Rep. 10, 701 (2020).

113. Topalian, S. L. et al. Safety, activity, and immune correlates of anti-PD-1 antibody in cancer. N. Engl. J. Med. 366, 2443-2454 (2012).

114. Shin, S. J. et al. Clinicopathologic analysis of PD-L1 and PD-L2 expression in renal cell carcinoma: association with oncogenic proteins status. Ann. Surg. Oncol. 23, 694-702 (2016)

115. Chen, L. Co-inhibitory molecules of the B7-CD28 family in the control of T-cell immunity. Nat. Rev. Immunol. 4, 336-347 (2004).

116. Weinstock, M. \& McDermott, D. Targeting PD-1/PD $\mathrm{L} 1$ in the treatment of metastatic renal cell carcinoma Ther. Adv. Urol. 7, 365-377 (2015).

117. Thompson, R. H. et al. Tumor B7-H1 is associated with poor prognosis in renal cell carcinoma patients with long-term follow-up. Cancer Res. 66, 3381-3385 (2006).

118. Herbst, R. S. et al. Predictive correlates of response to the anti-PD-L1 antibody MPDL3280A in cancer patients. Nature 515, 563-567 (2014).

119. Brahmer, J. R. et al. Safety and activity of anti-PD-L1 antibody in patients with advanced cancer. N. Engl. J. Med. 366, 2455-2465 (2012).

120. Thompson, R. H. et al. PD-1 is expressed by tumor-infiltrating immune cells and is associated with poor outcome for patients with renal cell carcinoma. Clin. Cancer Res. 13, 1757-1761 (2007)

121. Choueiri, T. K. et al. PD-L1 expression in nonclear-cell renal cell carcinoma. Ann. Oncol. 25, 2178-2184 (2014).

122. Joseph, R. W. et al. PD-1 and PD-L1 expression in renal cell carcinoma with sarcomatoid differentiation. Cancer Immunol. Res. 3, 1303-1307 (2015).

123. Mickisch, G. H., Garin, A., van Poppel, H., de Prijck, L. ¿ Sylvester, R. Radical nephrectomy plus interferonalfa-based immunotherapy compared with interferon alfa alone in metastatic renal-cell carcinoma: a randomised trial. Lancet 358, 966-970 (2001).

124. Flanigan, R. C. et al. Nephrectomy followed by interferon alfa- $2 \mathrm{~b}$ compared with interferon alfa- $2 \mathrm{~b}$ alone for metastatic renal-cell cancer. N. Engl. J. Med. 345, 1655-1659 (2001)

125. Aslam, M. Z. \& Matthews, P. N. Cytoreductive nephrectomy for metastatic renal cell carcinoma: a review of the historical literature and its role in the era of targeted molecular therapy. ISRN Urol. 2014, 717295 (2014).

126. Hanna, N. et al. Survival analyses of patients with metastatic renal cancer treated with targeted therapy with or without cytoreductive nephrectomy: a national cancer data base study. J. Clin. Oncol. 34, 3267-3275 (2016).

127. Petrelli, F. et al. Cytoreductive nephrectomy in metastatic renal cell carcinoma treated with targeted therapies: a systematic review with a meta-analysis. Clin. Genitourin. Cancer 14, 465-472 (2016).

128. Heng, D. Y. et al. Cytoreductive nephrectomy in patients with synchronous metastases from renal cell carcinoma: results from the International Metastatic Renal Cell Carcinoma Database Consortium. Eur. Urol. 66, 704-710 (2014).

129. Kutikov, A. et al. Use of systemic therapy and factors affecting survival for patients undergoing cytoreductive nephrectomy. BJU Int. 106, 218-223 (2010).

130. Thomas, A. Z. et al. The role of metastasectomy in patients with renal cell carcinoma with sarcomatoid dedifferentiation: a matched controlled analysis. J. Urol. 196, 678-684 (2016).

131. Motzer, R. J. \& Russo, P. Cytoreductive nephrectomy - patient selection is key. N. Engl. J. Med. 379 , 481-482 (2018)

132. Ljungberg, B. et al. EAU guidelines on renal cell carcinoma: 2014 update. Eur. Urol. 67, 913-924 (2015).

133. Deschavanne P J \& Fertil, B. A review of human cell radiosensitivity in vitro. Int. J. Radiat. Oncol. Biol. Phys. 34, 251-266 (1996)

134. Tunio, M. A., Hashmi, A. \& Rafi, M. Need for a new trial to evaluate postoperative radiotherapy in renal cell carcinoma: a meta-analysis of randomized controlled trials. Ann. Oncol. 21, 1839-1845 (2010)

135. van der Werf-Messing, B. Proceedings: carcinoma of the kidney. Cancer 32, 1056-1061 (1973).

136. Siva, S. et al. Radiotherapy for renal cell carcinoma: renaissance of an overlooked approach. Nat. Rev. Urol. 14, 549 (2017).

137. Eminaga, O., Akbarov, I., Wille, S. \& Engelmann, U. Does postoperative radiation therapy impact survival in non-metastatic sarcomatoid renal cell carcinoma? 
A SEER-based study. Int. Urol. Nephrol. 47 1653-1663 (2015)

138. Alt, A. L. et al. Survival after complete surgical resection of multiple metastases from renal cell carcinoma. Cancer 117, 2873-2882 (2011).

139. Dabestani, S. et al. Local treatments for metastases of renal cell carcinoma: a systematic review. Lancet Oncol. 15, e549-e561 (2014).

140. Correa, R. J. M. et al. The emerging role of stereotactic ablative radiotherapy for primary renal cell carcinoma: a systematic review and meta-analysis. Eur. Urol. Focus. (2019).

141. Staehler, M. et al. Single fraction radiosurgery for the treatment of renal tumors. J. Urol. 193, 771-775 (2015).

142. Frick, M. A., Chhabra, A. M., Lin, L. \& Simone, C. B. II, First ever use of proton stereotactic body radiation therapy delivered with curative intent to bilateral synchronous primary renal cell carcinomas. Cureus $\mathbf{9}$, e1799 (2017).

143. Golshayan, A. R. et al. Metastatic sarcomatoid renal cell carcinoma treated with vascular endothelial growth factor-targeted therapy. J. Clin. Oncol. 27 235-241 (2009)

144. Voss, M. H. et al. Treatment outcome with mTOR inhibitors for metastatic renal cell carcinoma with nonclear and sarcomatoid histologies. Ann. Oncol. 25, 663-668 (2014)

145. Pinedo, H. M. $\&$ Verweij, J. The treatment of soft tissue sarcomas with focus on chemotherapy: a review. Radiother. Oncol. 5, 193-205 (1986)

146. Culine, S., Bekradda, M. Terrier-Lacombe, M. J. \& Droz, J. P. Treatment of sarcomatoid renal cell carcinoma: is there a role for chemotherapy? Eur. Urol 27, 138-141 (1995).

147. Escudier, B. et al. Doxorubicin and ifosfamide in patients with metastatic sarcomatoid renal cell carcinoma: a phase II study of the Genitourinary Group of the French Federation of Cancer Centers. J. Urol. 168, 959-961 (2002)

148. Nanus, D. M., Garino, A., Milowsky, M. I., Larkin, M. \& Dutcher, J. P. Active chemotherapy for sarcomatoid and rapidly progressing renal cell carcinoma. Cancer 101, 1545-1551 (2004).

149. Haas, N. B. et al. A phase II trial of doxorubicin and gemcitabine in renal cell carcinoma with sarcomatoid features: ECOG 8802. Med. Oncol. 29, 761-767 (2012).

150. Dutcher, J. P. \& Nanus, D. Long-term survival of patients with sarcomatoid renal cell cancer treated with chemotherapy. Med. Oncol. 28, 1530-1533 (2011).

151. Staehler, M. et al. Sorafenib after combination therapy with gemcitabine plus doxorubicine in patients with sarcomatoid renal cell carcinoma: a prospective evaluation. Eur. J. Med. Res. 15, 287-291 (2010)

152. Kyriakopoulos, C. E et al. Outcome of patients with metastatic sarcomatoid renal cell carcinoma: results from the International Metastatic Renal Cell Carcinoma Database Consortium. Clin. Genitourin. Cancer 13, e79-e85 (2015).

153. Molina, A. M et al. Sarcomatoid-variant renal cell carcinoma: treatment outcome and survival in advanced disease. Am. J. Clin. Oncol. 34, 454-459 (2011).

154. Michaelson, M. D. et al. Phase 2 trial of sunitinib and gemcitabine in patients with sarcomatoid and/or poor-risk metastatic renal cell carcinoma. Cancer 121, 3435-3443 (2015).

155. Maiti, A. et al. Phase 2 trial of capecitabine gemcitabine, and bevacizumab in sarcomatoid renal-cell carcinoma. Clin. Genitourin. Cancer (2017).

156. Jonasch, E. et al. Treatment of metastatic renal carcinoma patients with the combination of gemcitabine, capecitabine and bevacizumab at a tertiary cancer centre. BJU Int. 107, 741-747 (2011)

157. Haas, N. B. et al. ECOG 1808: Randomized phase II trial of sunitinib with or without gemcitabine in advanced kidney cancer with sarcomatoid features. J. Clin. Oncol. 34, 4511-4511 (2016)

158. Achkar, T. et al. High-dose interleukin 2 in patients with metastatic renal cell carcinoma with sarcomatoid features. PLoS One 12, e0190084 (2017).

159. Figlin, R. et al. Interleukin-2-based immunotherapy for the treatment of metastatic renal cell carcinoma an analysis of 203 consecutively treated patients. Cancer J. Sci. Am. 3, S92-S97 (1997).
160. Rosenberg, S. A., Yang, J. C., White, D. E. \& Steinberg, S. M. Durability of complete responses in patients with metastatic cancer treated with high-dose interleukin-2: identification of the antigens mediating response. Ann. Surg. 228, 307-319 (1998).

161. Fyfe, G. et al. Results of treatment of 255 patients with metastatic renal cell carcinoma who received high-dose recombinant interleukin-2 therapy. J. Clin. Oncol. 13, 688-696 (1995)

162. Coppin, C. et al. Immunotherapy for advanced renal cell cancer. Cochrane Database Syst. Rev. https://doi. org/10.1002/14651858.CD001425. pub2 (2004).

163. Derosa, L. et al Safety of available treatment options for renal cell carcinoma. Expert Opin. Drug Saf. 15, 1097-1106 (2016)

164. Negrier, S. et al. Recombinant human interleukin-2, recombinant human interferon Alfa-2a, or both in metastatic renal-cell carcinoma. N. Engl. J. Med. 338, 1272-1278 (1998).

165. Amin, A. \& White, R. L. Interleukin-2 in renal cell carcinoma: a has-been or a still-viable option? J. Kidney Cancer VHL 1, 74-83 (2014).

166. Taube, J. M et al. Association of PD-1, PD-1 ligands, and other features of the tumor immune microenvironment with response to anti-PD-1 therapy. Clin. Cancer Res. 20, 5064-5074 (2014).

167. McGregor, B. A. et al. Results of a multicenter phase i study of atezolizumab and bevacizumab for patients with metastatic renal cell carcinoma with variant histology and/or sarcomatoid features. J. Clin. Oncol. 38, 63-70 (2020).

168. Rouvinov, K. et al. Rapid response to nivolumab in a patient with sarcomatoid transformation of chromophobe renal cell carcinoma. Clin. Genitourin. Cancer 15, e1127-e1130 (2017).

169. Geynisman, D. M. Anti-programmed cell death protein 1 (PD-1) antibody nivolumab leads to a dramatic and rapid response in papillary rnal cell carcinoma with sarcomatoid and rhabdoid features. Eur. Urol. 68 912-914 (2015).

170. El Mouallem, N. Smith, S. C \& Paul, A. K. Complete response of a patient with metastatic sarcomatoid renal cell carcinoma to a programmed death-1 checkpoint inhibitor. J. Oncol. Pract. 14, 511-513 (2018).

171. McDermott, D. F. et al. Atezolizumab, an antiprogrammed death-ligand 1 antibody, in metastatic renal cell carcinoma: long-term safety, clinical activity, and immune correlates from a phase la study. J. Clin. Oncol. 34, 833-842 (2016).

172. Motzer, R. J. et al. Nivolumab plus Ipilimumab versus sunitinib in advanced renal-cell carcinoma. N. Engl. J. Med. 378, 1277-1290 (2018).

173. Tannir, N. M. et al. Efficacy and safety of nivolumab plus ipilimumab versus sunitinib in first-line treatment of patients with advanced sarcomatoid renal cell carcinoma. Clin.Cancer Res. https://doi.org/10.1158/ 1078-0432.Ccr-20-2063 (2020)

174. McDermott DF, M. R. et al. CheckMate 214 retrospective analyses of nivolumab plus ipilimumab or sunitinib in IMDC intermediate/poor-risk patients with previously untreated advanced renal cell carcinoma with sarcomatoid features. Abstract presented at: The Seventeenth International Kidney Cancer Symposium;2-3, 2018 (American Society of Clinical Oncology, Miami, Florida, 2018)

175. Rini, B. I. et al. The Society for Immunotherapy of Cancer consensus statement on immunotherapy for the treatment of advanced renal cell carcinoma (RCC). J. ImmunoTherapy Cancer 7, 354 (2019).

176. Rini, B. I. et al. Pembrolizumab plus axitinib versus sunitinib for advanced renal-cell carcinoma. N. Engl. J. Med. 380, 1116-1127 (2019).

177. Rini, B. I. et al. Pembrolizumab (pembro) plus axitinib (axi) versus sunitinib as first-line therapy for metastatic renal cell carcinoma (mRCC): outcomes in the combined IMDC intermediate/poor risk and sarcomatoid subgroups of the phase 3 KEYNOTE-426 study. J. Clin. Oncol. 37, 4500-4500 (2019).

178. Eisenhauer, E. A. et al. New response evaluation criteria in solid tumours: revised RECIST guideline (version 1.1). Eur. J. Cancer 45, 228-247 (2009).

179. Motzer, R. J. et al. Avelumab plus axitinib versus sunitinib for advanced renal-cell carcinoma. N. Engl. J. Med. 380, 1103-1115 (2019).
180. Choueiri, T. K. et al. 910PD - Efficacy and biomarker analysis of patients (pts) with advanced renal cell carcinoma (aRCC) with sarcomatoid histology (SRCC): subgroup analysis from the phase III JAVELIN renal 101 trial of first-line avelumab plus axitinib $(A+A x)$ vs sunitinib (S). Ann. Oncol. 30, v361 (2019).

181. Rini, B. I. et al. Atezolizumab plus bevacizumab versus sunitinib for patients with untreated metastatic renal cell carcinoma and sarcomatoid features: a prespecified subgroup analysis of the IMmotion 151 clinical trial. Eur. Urol. https://doi.org/10.1016/ j.eururo.2020.06.021 (2020).

182. Rini, B. I. et al. Atezolizumab plus bevacizumab versus sunitinib in patients with previously untreated metastatic renal cell carcinoma (IMmotion 151): a multicentre, open-label, phase 3, randomised controlled trial. Lancet 393, 2404-2415 (2019).

183. lacovelli, R. et al. Patients with sarcomatoid renal cell carcinoma - re-defining the first-line of treatment: A meta-analysis of randomised clinical trials with immune checkpoint inhibitors. Eur. J. Cancer 136, 195-203 (2020).

184. US National Library of Medicine. ClinicalTrials.gov https://clinicaltrials.gov/show/NCT03483883 (2018).

185. US National Library of Medicine. ClinicalTrials.gov https://clinicaltrials.gov/show/NCT03793166 (2019).

186. Wing, K. et al. CTLA-4 control over Foxp3 ${ }^{+}$regulatory T cell function. Science 322, 271-275 (2008)

187. Griffiths, R. W. et al. Frequency of regulatory T cells in renal cell carcinoma patients and investigation of correlation with survival. Cancer Immunol. Immunother. 56, 1743-1753 (2007).

188. Kang, M. J. et al. Tumor-infiltrating PD1-positive lymphocytes and FoxP3-positive regulatory $\mathrm{T}$ cells predict distant metastatic relapse and survival of clear cell renal cell carcinoma. Transl. Oncol. 6, 282-289 (2013).

189. Liotta, F. et al. Frequency of regulatory $T$ cells in peripheral blood and in tumour-infiltrating lymphocytes correlates with poor prognosis in renal cell carcinoma. BJU Int. 107, 1500-1506 (2011).

190. Schvartsman, G., Carneiro, A, Filippi, R. Z., Rao, P. $\&$ Msaouel, P. Rapid deep responses with nivolumab plus ipilimumab in papillary renal cell carcinoma with sarcomatoid dedifferentiation. Clin. Genitourin. Cancer 17, 315-318 (2019)

191. Takahashi, T. et al. Immunologic self-tolerance maintained by $\mathrm{CD} 25^{+} \mathrm{CD}^{+}$regulatory T cells constitutively expressing cytotoxic T lymphocyteassociated antigen 4. J. Exp. Med. 192, 303-310 (2000).

192. Al-Ahmadie, H. A. et al. Carbonic anhydrase IX expression in clear cell renal cell carcinoma: an immunohistochemical study comparing 2 antibodies. Am. J. Surg. Pathol. 32, 377-382 (2008).

\section{Author contributions}

K.A.B. researched data for the article. K.A.B., S.G., J.A.K. and A.A.H. wrote the article. All authors made substantial contributions to discussion of the content and reviewed/edited the manuscript before submission.

\section{Competing interests}

J.A.K. is a member of consulting or advisory boards for, and has received honoraria from, Merck, EMD Serono, Genentech, Novartis and Pfizer. J.A.K. has received institutional research funding from Merck, Genentech and Mirati. R.J.M. has received grants and paid consultancy from Eisai, Exelixis, Genentech, Roche, Novartis, Merck and Pfizer, paid consultancy from Astra Zeneca and grants from Bristol Myers Squibb. All other authors declare no competing interests.

\section{Peer review information}

Nature Reviews Urology thanks G. Malouf, M. Staehler and the other, anonymous, reviewer(s) for their contribution to the peer review of this work.

\section{Publisher's note}

Springer Nature remains neutral with regard to jurisdictional claims in published maps and institutional affiliations.

\section{Supplementary information}

Supplementary information is available for this paper at https://doi.org/10.1038/s41585-020-00382-9.

(C) Springer Nature Limited 2020 